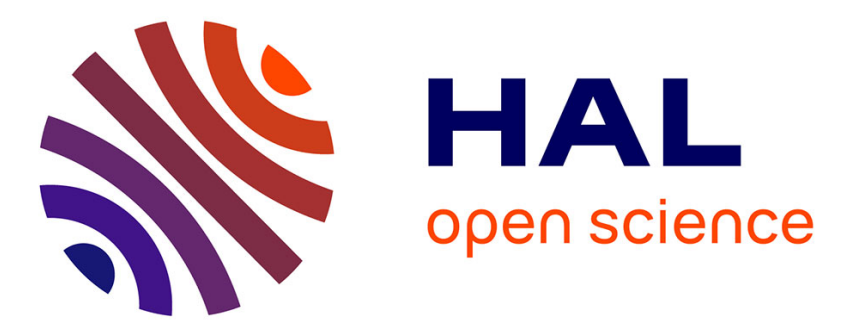

\title{
Development of noncentered wavenumber-based optimized interpolation schemes with amplification control for overlapping grids
}

Damien Desvigne, Olivier Marsden, Christophe Bogey, Christophe Bailly

\section{- To cite this version:}

Damien Desvigne, Olivier Marsden, Christophe Bogey, Christophe Bailly. Development of noncentered wavenumber-based optimized interpolation schemes with amplification control for overlapping grids. SIAM Journal on Scientific Computing, 2010, 32, pp.2074-2098. 10.1137/090758702 . hal-00566011

\section{HAL Id: hal-00566011 \\ https://hal.science/hal-00566011}

Submitted on 8 Jun 2012

HAL is a multi-disciplinary open access archive for the deposit and dissemination of scientific research documents, whether they are published or not. The documents may come from teaching and research institutions in France or abroad, or from public or private research centers.
L'archive ouverte pluridisciplinaire $\mathbf{H A L}$, est destinée au dépôt et à la diffusion de documents scientifiques de niveau recherche, publiés ou non, émanant des établissements d'enseignement et de recherche français ou étrangers, des laboratoires publics ou privés. 


\title{
DEVELOPMENT OF NONCENTERED WAVENUMBER-BASED OPTIMIZED INTERPOLATION SCHEMES WITH AMPLIFICATION CONTROL FOR OVERLAPPING GRIDS*
}

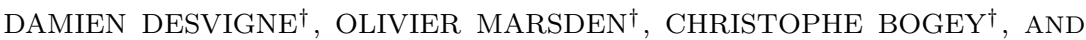 \\ CHRISTOPHE BAILLY ${ }^{\dagger}$
}

\begin{abstract}
A noncentered interpolation technique has been constructed to perform simulations using overlapping grids for complex geometries. High-order centered Lagrange polynomial interpolations and interpolations optimized in the Fourier space are first generalized to the noncentered case. These noncentered interpolations either generate significant dispersion errors or strongly amplify high-wavenumber components. Accordingly, a noncentered high-order wavenumber-based optimized interpolation method is developed with the addition of a nonlinear constraint for the control of the amplitude amplification induced by decentering. High-order piecewise polynomial regressions of the obtained interpolation coefficients are performed. The time stability of the method is investigated in the 1-D case when the interpolation method is used in conjunction with explicit high-order differencing, filtering schemes, as well as a 6-step Runge-Kutta time integration algorithm. A criterion is formulated to predict its stability as a function of the filtering strength and the Courant-FriedrichsLewy constant. Finally, 1-D convection simulations are presented to illustrate the stability and the accuracy of the developed noncentered interpolations.
\end{abstract}

Key words. noncentered interpolations, decentering, high order, overlapping grids, Fourier space

AMS subject classifications. 03C40, 41A05, 65D05

DOI. $10.1137 / 090758702$

1. Introduction. The field of computational aeroacoustics (CAA) has been widely expanded over the past twenty years with the increasing need from aeronautic industries for predictive computations [10]. One new aspect of CAA is the direct computation of aerodynamic noise, namely the unsteady turbulent field and the noise radiated by the flow [5]. The disparity of scales that must be resolved - several orders of magnitude separate the energy and wavelengths of propagative acoustic fluctuations from those of the flow features - entails strong constraints on the numerical methods. This can be illustrated by the development of high-order schemes to solve CAA governing equations, such as Tam and Webb's dispersion-relation-preserving (DRP) finite-difference schemes [33], Lele's compact finite-difference schemes [19], or, more recently, Bogey and Bailly's schemes [5]. All these numerical methods are centered in space and nondissipative. In addition, some selective filters have been developed to remove nonresolved short-wavelength contributions to the numerical solution, hence preserving the computation's accuracy for longer wavelengths [5]. Investigations for tractable formulations of accurate numerical boundary conditions are other illustrations of the recent development of precise numerical methods in CAA [3, 31].

The difficulty in achieving an accurate computation can also come from the complexity of geometries. Complex geometries - while easy to handle thanks to a non-

*Received by the editors May 11, 2009; accepted for publication (in revised form) April 26, 2010; published electronically July 20, 2010. This work was supported by the Fondation de Recherche pour l'Aéronautique \& l'Espace (FRAE) under contract reference AFB AEROCAV.

http://www.siam.org/journals/sisc/32-4/75870.html

${ }^{\dagger}$ Laboratoire de Mécanique des Fluides et d'Acoustique, UMR CNRS 5509 - Centre Acoustique, École Centrale de Lyon, 36 avenue Guy de Collongue, 69134 Écully Cedex, France (damien.desvigne@ ec-lyon.fr, olivier.marsden@ec-lyon.fr, christophe.bogey@ec-lyon.fr, christophe.bailly@ec-lyon.fr).

2074 
structured method - are in most cases impossible to mesh with a single structured grid [22]. Nevertheless, those difficulties can be overcome by the use of overlapping grid methods, also referred to as chimera methods [2] or multidomain methods [21]. These techniques have aroused a certain interest over the last twenty years [12, 13, 28, 32] and recent simulations have shown that it is possible to compute acoustic phenomena on multidomains with high fidelity. Thus, Marsden, Bogey, and Bailly [22] used a chimera method to simulate the diffraction of sound by two cylinders of different diameters in a low Mach number flow. Lummer, Delfs, and Lauke [20] implemented multidomain techniques to investigate interactions between vortices and the trailing edge of airfoils in the noise generation. Freund, Lele, and Moin [14] suggested resorting to a Cartesian-cylindrical multidomain to solve CAA equations for a cylindrical geometry: the annulus is cylindrically meshed, whereas the core grid is Cartesian. The purpose of this approach is to avoid the axis singularity of cylindrical Navier-Stokes equations.

The communication between the different grids of the computational domain is a key element for the successful achievement of an accurate numerical simulation. The use of high-order polynomials is by far the most popular method for interpolations in the mesh-to-mesh transfer of the solution [32]. Tam and Kurbatskii developed in [32] an optimized interpolation by minimizing the interpolation integral error. Other optimized interpolations have also been developed over the last decades, such as Sherer and Scott's implicit optimized interpolation [28]. However, all these methods need a large centered interpolation stencil to be sufficiently accurate - typically a 6-to12-point stencil - and as a consequence cannot be directly used for interpolations at mesh nodes that are close to a wall for geometric reasons. This situation may occur in some cases: for instance, in the investigation of the sound induced by a flow over a cylindrical cavity, noncentered interpolations greatly simplify communication between the different grids in the cavity opening, or lip region, as illustrated in Figure 1. Numerical techniques resorting to a ghost-node method have been developed to use centered schemes even in the near-wall region [16, 31], but some difficulties remain regarding how to determine the values at ghost nodes. Noncentered interpolations may be another solution to consider, in order to cope with interpolation problems at walls. Noncentered Lagrange and least-square polynomial interpolations are studied by Peller et al. for immersed boundary methods [25], but their work focused on stability without examining interpolation errors. Within the context of CAA simulations, noncentered interpolations are used in conjunction with a finite-difference scheme for derivative approximations and a selective filter. Thus, interpolation errors must be controlled to maintain comparable accuracy with respect to the errors induced by the chosen finite-difference scheme and the selective filter. In addition, noncentered interpolations are known to be strong high-wavenumber amplifiers [25, 32], which must be controlled as well to stabilize simulations. These constraints consequently establish severe criteria in the choice of suitable noncentered interpolation methods.

In this article we focus on noncentered high-order interpolations, and describe the construction of a high-order noncentered interpolation method for which highwavenumber amplification is controlled. Results for 1-D test cases are also presented. For the sake of simplicity, we will discuss only 1-D interpolations here. This restriction entails no loss of generality, because it has been shown that multidimensional interpolations can be performed using tensor products of 1-D interpolation basis sets, which reduces multidimensional interpolations to a sequence of 1-D interpolations $[7,9,21]$. 
(a)

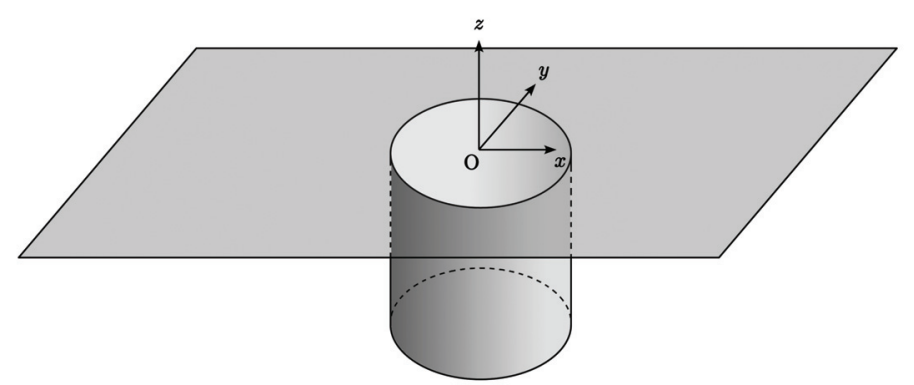

(b)

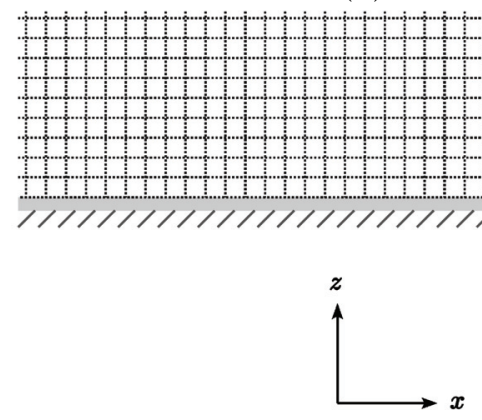

$(\mathrm{c})$

(c)

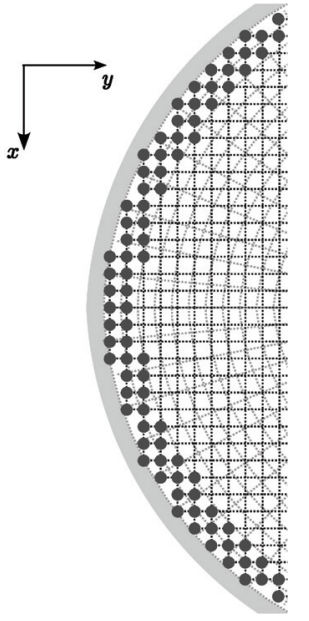

FIG. 1. Illustration of the use of overlapping grids for the simulation of a cavity flow. (a): Cavity overview. (b): Multidomain transversal view in the plane $y=0$. (c): Multidomain innercavity view in the lip zone at a constant $z$. Cavity annulus is cylindrically meshed (in gray). Top grid is Cartesian (in black): top-grid nodes requiring noncentered interpolation from annulus grid are highlighted in black.

2. Generalization of centered interpolations to the noncentered case. High-order polynomial centered interpolations and wavenumber-based optimized centered interpolations have proven their efficiency in direct computations of noise induced by turbulent flows over bodies $[15,18,21]$. A generalization of those methods to the noncentered case is considered here.

2.1. Mathematical background. Let $n$ be an integer in $\mathbb{N}^{*} \backslash\{1\}$. Let $\mathcal{S}=$ $\left\{x_{0}, x_{1}, \ldots, x_{n-1}\right\}$ be an ordered set of $n$ real numbers verifying $x_{j}=x_{0}+j \Delta x$, for all $j \in\{0,1, \ldots, n-1\}$, with $\Delta x$ the grid spacing $(\Delta x>0)$. This 1-D interpolation stencil of donor points is shown in Figure 2. The set of $n$ points defines $n-1$ subintervals where interpolations can be performed at an arbitrary point $x \in\left[x_{0}, x_{n-1}[\right.$. In the following, we assume interpolations are performed at the $d$ th cell of the stencil $(d \in$ $\{1, \ldots, n-1\})$, as depicted in Figure 2, so that $x=x_{0}+(d-1+\eta) \Delta x$, where $\eta$ is the fractional part of $\left(x-x_{0}\right) / \Delta x(\eta \in[0,1[)$. Note that the mesh step $\Delta x$ can be assumed to be uniform without loss of generality, which is justified by specifying that interpolations can be performed in the transformed computational space of the donating grid rather than in the physical space [29]. Let $f$ be a known discrete function from $\mathcal{S}$ to $\mathbb{C}$. One notes $f_{j}=f\left(x_{j}\right)$ for all $j \in\{0,1, \ldots, n-1\}$. Its interpolated value 


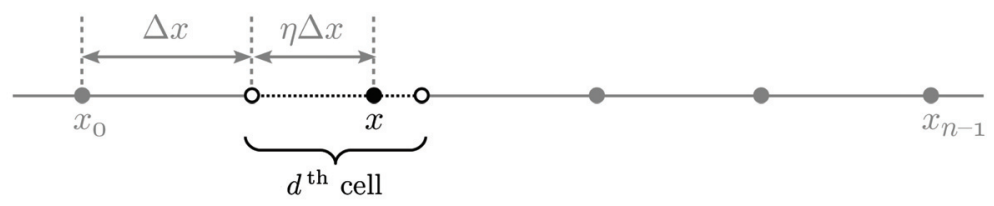

FIG. 2. Noncentered dth cell interpolation stencil, with $n=6$ and $d=2$.

at a receiver point $x$ is noted $\widetilde{f}(x)$. According to Sherer and Scott [29], a noncoupled explicit 1-D interpolation formulation can be written as

$$
\forall x \in\left[x_{d-1}, x_{d}\left[, \quad \tilde{f}(x)=\sum_{j=0}^{n-1} S_{j}(x) f_{j},\right.\right.
$$

where $S_{j}(x)$ are $n$ interpolation coefficients. In what follows, one notes $s_{j}^{d}(\eta)=$ $S_{j}(x)=S_{j}\left(x_{0}+(d-1+\eta) \Delta x\right)$ and $\chi=-(d-1+\eta)$. Equation (1) can be reformulated as

$$
\forall \eta \in\left[0,1\left[, \quad \widetilde{f}(x)=\sum_{j=0}^{n-1} s_{j}^{d}(\eta) f_{j} .\right.\right.
$$

Thus, an explicit $d$ th cell $n$-point interpolation method is fully determined by the construction of the $n$ functions $s_{j}^{d}$, defined from $[0,1[$ to $\mathbb{R}$. In addition, it can be noted that an explicit $(n-d)$ th cell $n$-point interpolation method can be deduced from the explicit $d$ th cell $n$-point interpolation thanks to the reflection formula

$$
s_{j}^{n-d}(\eta)=s_{n-1-j}^{d}(1-\eta) .
$$

2.2. Noncentered Lagrange polynomial interpolations. For all $x_{j}$ in $\mathcal{S}$, a unique polynomial $\ell_{j}$ of maximum degree $n-1$, with a value of 1 at $x_{j}$ and 0 at all other stencil nodes, can be constructed [17, 34]:

$$
\forall x \in\left[x_{0}, x_{n-1}\left[, \quad \ell_{j}(x)=\prod_{q=0, q \neq j}^{n-1} \frac{x-x_{q}}{x_{j}-x_{q}} .\right.\right.
$$

The interpolated value of $f$ at $x \in\left[x_{d-1}, x_{d}[\right.$ is then given by

$$
\forall x \in\left[x_{d-1}, x_{d}\left[, \quad \widetilde{f}(x)=\sum_{j=0}^{n-1} \ell_{j}(x) f_{j} .\right.\right.
$$

From (2) and (3), a projection on the canonical orthogonal base of vector space $\mathcal{S} \mapsto \mathbb{C}$ gives

$$
s_{j}^{d}(\eta)=\prod_{q=0, q \neq j}^{n-1} \frac{q+\chi}{q-j} .
$$

In the present article, noncentered Lagrange interpolations are referred to as LIn $\mathrm{p}^{d}$, where $n$ is the number of points of the interpolation stencil, and $d$ corresponds to the stencil cell where interpolations are performed. Note that the 2-point linear interpolation is defined by considering the 2-point centered Lagrange polynomial interpolation LI2 $\mathrm{p}^{1}$. 
Interpolation error quantification. The noncentered local interpolation error can be built from the formulation proposed by Tam and Kurbatskii [32]. Let $f_{k}$ be a harmonic test function of wavenumber $k$ and phase $\phi$ :

$$
f_{k}:\left\{\begin{array}{clc}
{\left[x_{0}, x_{n-1}\right]} & \longmapsto & \mathbb{C} \\
x & \longmapsto & e^{i(k x+\phi)} \quad(\phi \in[-\pi, \pi[) .
\end{array}\right.
$$

The $d$ th cell interpolation local error $\varepsilon_{\text {loc }}^{d}(\eta, k \Delta x)$ can be defined for all $x \in\left[x_{d-1}, x_{d}[\right.$ as

$$
\varepsilon_{\mathrm{loc}}^{d}(\eta, k \Delta x)=\left|\frac{f_{k}(x)-\widetilde{f}_{k}(x)}{f_{k}(x)}\right|=\left|1-\sum_{j=0}^{n-1} s_{j}^{d}(\eta) e^{i(j+\chi) k \Delta x}\right| .
$$

The $d$ th cell interpolation global error $\varepsilon^{d}(k \Delta x)$ is expressed as

$$
\varepsilon^{d}(k \Delta x)=\max _{\eta \in[0,1[} \varepsilon_{\text {loc }}^{d}(\eta, k \Delta x)
$$

Figure 3 shows the distribution of 1 st cell interpolation error $\varepsilon^{1}$ in the wavenumber space for different noncentered Lagrange interpolations, using stencils from 2 to 10 points in length. For low wavenumbers, increasing the size of the interpolation stencil clearly makes the error drop. At the same time, the interpolation error for the high wavenumbers increases dramatically. Note that the linear interpolation using a 2-point stencil LI2 $\mathrm{p}^{1}$ may not be accurate enough even for low wavenumbers. Indeed, LI $2 \mathrm{p}^{1}$ interpolation error is several orders of magnitude larger than the error introduced by other numerical methods with which it is supposed to be used, such as high-order finite differences or selective filtering. Its interpolation error, which should be smaller than $10^{-4}$ for $k \Delta x \leq \pi / 8$ and smaller than $10^{-5}$ for $k \Delta x \leq \pi / 16$ in order to be coherent with other parts of the numerical scheme, is in fact greater than $10^{-3}$ for $k \Delta x \geq \pi / 32$.

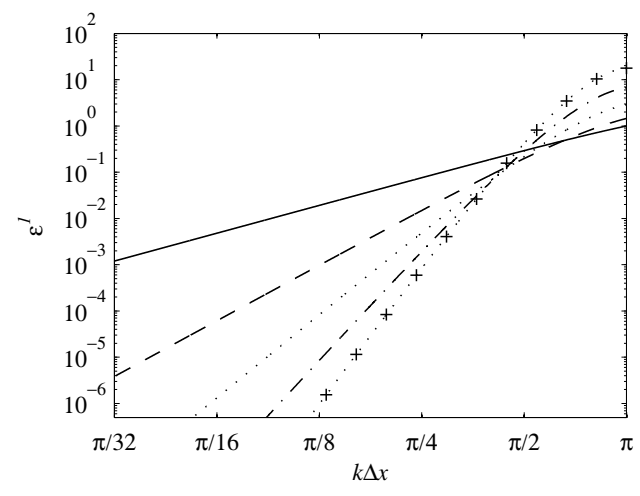

FIG. 3. First-cell interpolation error $\varepsilon^{1}$ as a function of $k \Delta x$ for different stencil sizes of Lagrange interpolations. $-\mathrm{LI}_{2} \mathrm{p}^{1},---\mathrm{LI} 4 \mathrm{p}^{1}, \cdots \cdots \mathrm{LI}^{1} \mathrm{p}^{1},-\cdots \mathrm{LI}^{1} \mathrm{p}^{1}, \cdots+\cdots \quad$ LI10p ${ }^{1}$.

Amplification and phase error quantifications. The $d$ th cell local interpolation amplification $\alpha_{\text {loc }}^{d}(\eta, k \Delta x)$ can be calculated for all $x \in\left[x_{d-1}, x_{d}[\right.$ as

$$
\alpha_{\mathrm{loc}}^{d}(\eta, k \Delta x)=\left|\frac{\widetilde{f}_{k}(x)}{f_{k}(x)}\right|=\left|\sum_{j=0}^{n-1} s_{j}^{d}(\eta) e^{i(j+\chi) k \Delta x}\right| .
$$

Copyright $\odot$ by SIAM. Unauthorized reproduction of this article is prohibited. 
The $d$ th cell interpolation global amplification $\alpha^{d}(k \Delta x)$ is expressed as

$$
\alpha^{d}(k \Delta x)=\max _{\eta \in[0,1[} \alpha_{\text {loc }}^{d}(\eta, k \Delta x) .
$$

In the same way, the $d$ th cell interpolation local phase error $\phi_{\text {loc }}^{d}(\eta, k \Delta x)$ can be estimated for all $x \in\left[x_{d-1}, x_{d}[\right.$ with the following equation:

$$
\phi_{\text {loc }}^{d}(\eta, k \Delta x)=\arg \frac{\widetilde{f}_{k}(x)}{f_{k}(x)}=2 \tan ^{-1}\left(-\frac{\mathfrak{R}}{\mathfrak{I}}+\operatorname{sgn}(\mathfrak{I}) \sqrt{1+\left(\frac{\mathfrak{R}}{\mathfrak{I}}\right)^{2}}\right),
$$

where $\mathfrak{R}$ and $\mathfrak{I}$ are, respectively, the real and imaginary parts of the complex number $\sum_{j=0}^{n-1} s_{j}^{d}(\eta) e^{i(j+\chi) k \Delta x}$. The $d$ th cell interpolation global phase error $\phi^{d}(k \Delta x)$ is defined as

$$
\phi^{d}(k \Delta x)=\max _{\eta \in[0,1[}\left|\phi_{\text {loc }}^{d}(\eta, k \Delta x)\right| .
$$

Figure 4(a) exhibits the amplification factor $\alpha^{1}$ in the wavenumber space for Lagrange interpolations using stencils from 2 to 10 points, and performed on the first cell. For low wavenumbers, the amplification is very close to 1 , but it increases significantly for high wavenumbers, and reaches a maximum for grid-to-grid oscillations. This phenomenon is exacerbated by an increase of the interpolation stencil size. Such a high-wavenumber amplification should exclude the use of this kind of noncentered interpolations in CAA. Indeed, the high-wavenumber selective damping might not be sufficient to offset such an interpolation amplification level, leading to a global pollution of numerical solutions from high-wavenumber instabilities. Figure 4(b) shows the phase error in the wavenumber space for first-cell Lagrange interpolations using stencils from 2 to 10 points. For all tested stencil sizes, phase error is very small for low wavenumbers, but it increases rapidly for $k \Delta x \geq \pi / 2$. A global maximum is obtained for grid-to-grid oscillations. A local maximum is also observed for high wavenumbers for 8-and-10-point interpolation stencils. It should be noted that other noncentered polynomial interpolations could be expressed, depending on different choices of polynomial basis. Boyd showed [8], for instance, that it can be relevant to construct the $s_{j}^{d}$ functions with Chebyshev polynomial basis sets. In the same way, this can be generalized to other nonpolynomial function spaces. Boyd thus proved that sinc-based interpolations could also be constructed [8].

2.3. Noncentered optimized interpolations. Noncentered Lagrange polynomial interpolations present one strong weakness: they behave as high-wavenumber amplifiers. It is therefore relevant to investigate optimized interpolations to overcome the latter failure. Tam and Kurbatskii proposed wavenumber-based optimized interpolations that are very accurate in the centered case [32]. The method is revisited for the noncentered case in the present subsection. Let $\varepsilon_{\text {int }}^{d}$ be the interpolation integral error at a location $\eta$ in the $d$ th interpolation cell, in the nondimensional wavenumber range $\left[\kappa_{l}, \kappa_{u}\right]$ :

$$
\varepsilon_{\mathrm{int}}^{d}(\eta)=\int_{\kappa_{l}}^{\kappa_{u}}\left(\varepsilon_{\mathrm{loc}}^{d}(\eta, k \Delta x)\right)^{2} d(k \Delta x) .
$$

This integral error is to be minimized in order to increase the interpolation accuracy limit. The choice of the integration boundaries is intrinsically linked to the context in 
(a)

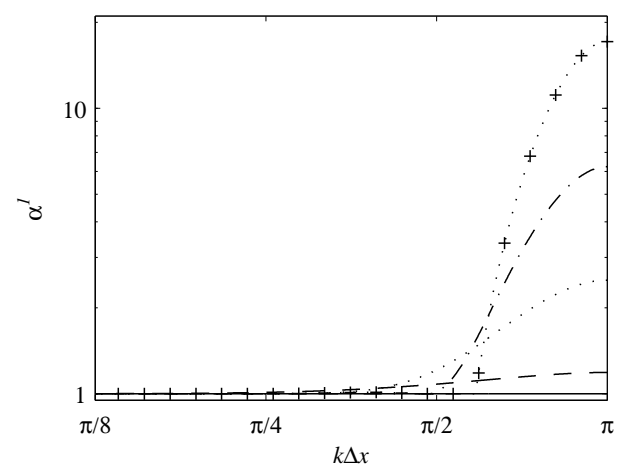

(b)

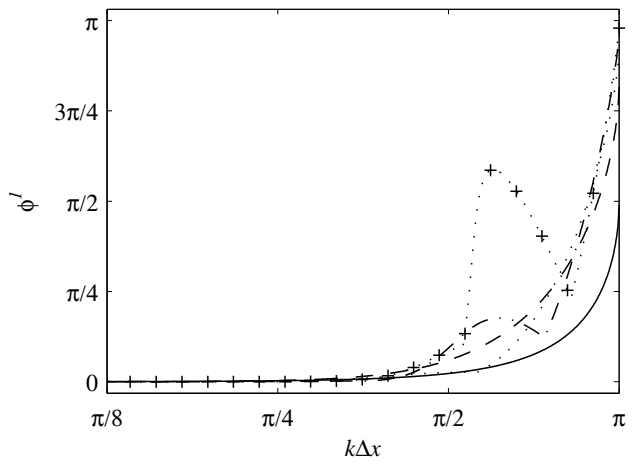

FIG. 4. (a): First-cell interpolation amplification $\alpha^{1}$ as a function of $k \Delta x$ for different stencil sizes of Lagrange interpolations. (b): First-cell interpolation phase error $\phi^{1}$ as a function of $k \Delta x$ for different stencil sizes of Lagrange interpolations. $-\mathrm{LI} 2 \mathrm{p}^{1},---\mathrm{LI} 4 \mathrm{p}^{1}, \cdots \cdots \mathrm{LI}^{1} \mathrm{p}^{1}$, ---LI8p ${ }^{1}, \cdots+\cdot$ LI10 ${ }^{1}$.

which the noncentered interpolations are used. In conjunction with high-order finitedifference schemes, $\kappa_{l}$ is taken as 0 and $\kappa_{u}$ as the accuracy limit of the considered finite-difference scheme. In our case, we choose to take $\kappa_{u}$ as the 11-point Bogey and Bailly optimized finite-difference scheme accuracy limit, i.e., $2 \pi / 5$ [5]. In this work, the resolved wavenumber range is defined as wavenumbers verifying $k \Delta x \in\left[\kappa_{l}, \kappa_{u}\right]$. In what follows, the quantity $\widetilde{f}$, which is the interpolation of the discrete function $f$, is assumed to be $\mathcal{C}^{n-1}$ in the open interval $] x_{0}, x_{n-1}[$. The optimized interpolation method is of order $p(p \in\{1,2, \ldots, n-1\})$. Thus, a $p$ th order Taylor expansion is performed for $\widetilde{f}\left(x_{j}\right)=f_{j}+\mathcal{O}\left(\Delta x^{p}\right)$ at location $x=x_{0}-\chi \Delta x$ :

$$
\forall j \in\{0,1, \ldots, n-1\}, \quad f_{j}=\sum_{r=0}^{p-1} \frac{(j+\chi)^{r} \Delta x^{r}}{r !} \widetilde{f}^{(r)}\left(x_{0}-\chi \Delta x\right)+\mathcal{O}\left(\Delta x^{p}\right) .
$$

These $n$ equations (6) are then reinjected into (2) and all terms of order $r \in\{1,2, \ldots, p-$ $1\}$ are canceled, giving the following $p$ equations:

$$
\forall r \in\{0,1, \ldots, p-1\}, \quad \sum_{j=0}^{n-1} s_{j}^{d}(\eta)(j+\chi)^{r}=\delta_{0}^{r},
$$

where $\delta_{0}^{r}$ is the Kronecker delta of the two integers 0 and $r$. These $p$ equations are consequently order constraints of the minimization problem of $\varepsilon_{\text {int }}^{d}$. The constrained optimization problem can be handled by the method of Lagrange multipliers [4]. Hence, $p$ Lagrange multipliers $\lambda_{r}$ are introduced, and the previous constrained optimization problem can be treated as a nonconstrained optimization problem for the Lagrangian function $\mathcal{L}$ defined as

$$
\begin{aligned}
\mathcal{L}\left(s_{0}^{d}, s_{1}^{d}, \ldots, s_{n-1}^{d}, \lambda_{0}, \ldots, \lambda_{p-1}\right) & =\int_{\kappa_{l}}^{\kappa_{u}}\left(\varepsilon_{\mathrm{loc}}^{d}(\eta, k \Delta x)\right)^{2} d(k \Delta x) \\
& +\sum_{r=0}^{p-1} \lambda_{r}\left(\sum_{j=0}^{n-1} s_{j}^{d}(j+\chi)^{r}-\delta_{0}^{r}\right) .
\end{aligned}
$$

Copyright $@$ by SIAM. Unauthorized reproduction of this article is prohibited. 
Interpolation coefficients $s_{j}^{d}$ and Lagrange multipliers $\lambda_{r}$ are found by solving the following linear system: $\forall l \in\{0,1, \ldots, n-1\}$ and $\forall r \in\{0,1, \ldots, p-1\}$,

$$
\frac{\partial \mathcal{L}}{\partial s_{l}^{d}}=0, \quad \frac{\partial \mathcal{L}}{\partial \lambda_{r}}=0 .
$$

This leads to the matrix equation

$$
\underbrace{\left[\begin{array}{cc}
\mathcal{M}_{1} & \mathcal{M}_{2} \\
{ }^{t} \mathcal{M}_{2} & 0
\end{array}\right]}_{\mathcal{M}} \cdot\left[\begin{array}{c}
s_{0}^{d}(\eta) \\
s_{1}^{d}(\eta) \\
\vdots \\
s_{n-1}^{d}(\eta) \\
\lambda_{0}(\eta) \\
\lambda_{1}(\eta) \\
\vdots \\
\lambda_{p-1}(\eta)
\end{array}\right]=\left[\begin{array}{c}
\mathcal{V} \\
1 / 2 \\
0 \\
\vdots \\
0
\end{array}\right],
$$

where the block matrix $\mathcal{M}$ is the $(n+p) \times(n+p)$ nonsingular symmetric matrix defined as

$$
\begin{aligned}
& \forall(l, j) \in\{0, \ldots, n-1\}^{2}, \quad \mathcal{M}_{1}(l+1, j+1)=\kappa_{u} \operatorname{sinc} \kappa_{u}(j-l)-\kappa_{l} \text { sinc } \kappa_{l}(j-l), \\
& \forall(l, j) \in\{0, \ldots, n-1\} \times\{0, \ldots, p-1\}, \quad \mathcal{M}_{2}(l+1, j+1)=1 / 2(l+\chi)^{j},
\end{aligned}
$$

and the vector $\mathcal{V}$ is expressed as

$$
\forall l \in\{0, \ldots, n-1\}, \quad \mathcal{V}(l+1)=\kappa_{u} \operatorname{sinc} \kappa_{u}(l+\chi)-\kappa_{l} \operatorname{sinc} \kappa_{l}(l+\chi) .
$$

The function sinc is the sine cardinal function:

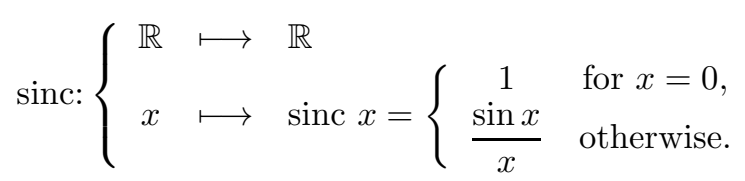

The solution is explicitly expressed thanks to Strassen's block inversion method [30]:

$$
\left[\begin{array}{c}
s_{0}^{d}(\eta) \\
s_{1}^{d}(\eta) \\
\vdots \\
s_{n-1}^{d}(\eta) \\
\lambda_{0}(\eta) \\
\lambda_{1}(\eta) \\
\vdots \\
\lambda_{p-1}(\eta)
\end{array}\right]=\left[\begin{array}{cc}
\mathcal{M}_{1}^{-1}+\mathcal{D C}^{-1}{ }^{t} \mathcal{D} & -\mathcal{D C}^{-1} \\
-\mathcal{C}^{-1}{ }^{t} \mathcal{D} & \mathcal{C}^{-1}
\end{array}\right] \cdot\left[\begin{array}{c}
\mathcal{V} \\
1 / 2 \\
0 \\
\vdots \\
0
\end{array}\right]
$$

where $\mathcal{C}$ is the Schur complement of the matrix $\mathcal{M}_{1}$ defined by $\mathcal{C}=-{ }^{t} \mathcal{M}_{2} \mathcal{D}$, where $\mathcal{D}=\mathcal{M}_{1}^{-1} \mathcal{M}_{2}$.

Properly speaking, it must be observed that these methods are not interpolations but approximations, because collocation conditions $f_{j}=\widetilde{f}\left(x_{j}\right)$ are no longer

Copyright $@$ by SIAM. Unauthorized reproduction of this article is prohibited. 
imposed and more flexible conditions are preferred: $f_{j}=\tilde{f}\left(x_{j}\right)+\mathcal{O}\left(\Delta x^{p}\right)$. Note that for Lagrange polynomial interpolations, collocation conditions are verified by construction. In the following, noncentered optimized interpolations are referred to as OInp $p \mathrm{o}^{d}$, where $n$ is the interpolation stencil size, $p$ the formal order of the interpolation method, and $d$ corresponds to the stencil cell where the interpolations are performed.

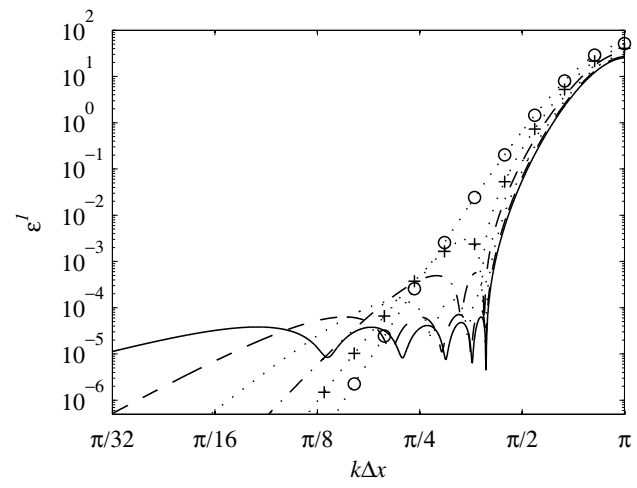

FIG. 5. First-cell interpolation error $\varepsilon^{1}$ as a function of $k \Delta x$ for different formal orders of optimized interpolations with a 12-point stencil and for the 12-point Lagrange interpolation.

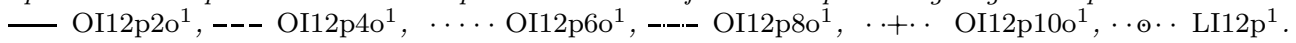

Figure 5 presents the distribution of first-cell interpolation error $\varepsilon^{1}$ in the Fourier space for different noncentered 12-point optimized interpolations for formal orders from 2 to 10. A comparison with the 12-point Lagrange interpolation LI12 ${ }^{1}$ shows that the error obtained for optimized interpolations is several orders of magnitude smaller in the wavenumber range $[\pi / 4, \pi / 2]$. In the low wavenumbers, the optimized interpolation error is higher than the Lagrange polynomial interpolation error, but remains below $5 \times 10^{-5}$ for 12-point optimized interpolation. Figure 6(a) displays the corresponding interpolation amplification. The amplification, which still occurs for optimized interpolations at very high wavenumbers, is maximum for grid-to-grid oscillations. The amplification factor increases significantly with an increase of the interpolation's formal order. Figure 6(b) exhibits the phase error for the 12-point optimized interpolations. The phase error remains close to 0 for low wavenumbers, but it increases significantly from around $k \Delta x=\pi / 2$ to reach the maximum phase error $\pi$ on a wavenumber interval. This is due to the fact that we considered here the maximum of the phase error in the whole interpolation cell (see (4)). In the wavenumber interval where the maximum is reached, it is observed that there always exists a location where a phase opposition occurs. Then the phase error decreases and increases again to reach $\pi$ for grid-to-grid oscillations.

Thus, a reduction of the interpolation's formal order leads to a significant improvement of the interpolation method, compared to noncentered Lagrange polynomial interpolations, as interpolation error remains low in the wavenumber range $[\pi / 4,2 \pi / 5]$ for noncentered optimized interpolations. Nevertheless, noncentered optimized interpolations may not be very suitable for numerical simulations because the wavenumber-based optimization does not fix the problem of the strong amplification for the high wavenumbers. High-wavenumber instabilities remain consequently amplified and may lead to unsatisfactory simulation results. 
(a)

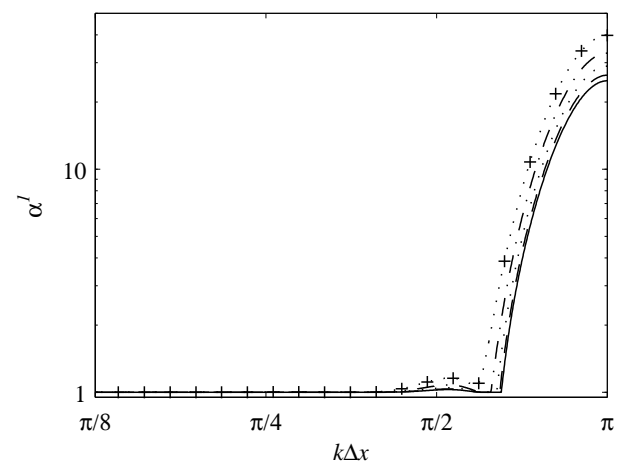

(b)

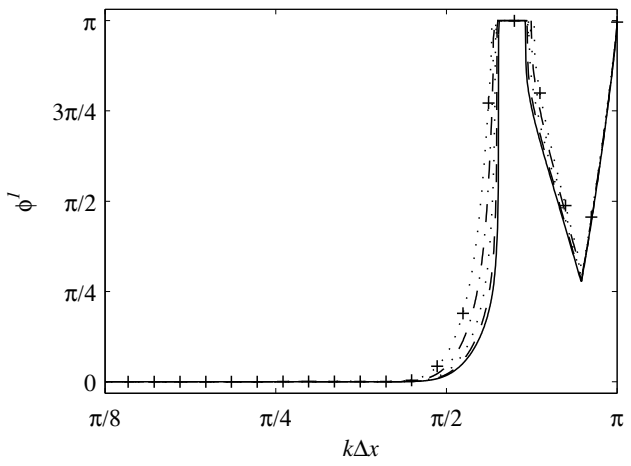

Fig. 6. (a): First-cell interpolation amplification $\alpha^{1}$ as a function of $k \Delta x$ for different formal orders of 12-point optimized interpolations. (b): First-cell interpolation phase error $\phi^{1}$ as a function of $k \Delta x$ for different formal orders of 12-point optimized interpolations. - - OI12p20 $\mathrm{p}^{1}$, ---

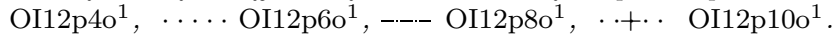

3. Noncentered optimized interpolations with amplification control. In practical cases, it has been seen that the interpolation amplification can be up to two orders of magnitude greater than 1, and reaches a maximum for grid-to-grid oscillations. This is a severe drawback for the stability of high-fidelity simulations. In this section, this problem of grid-to-grid amplification is handled with the addition of an extra constraint in the construction of noncentered wavenumber-based optimized interpolation methods.

A local formulation of the amplification control is expressed. The amplification is limited $1+\alpha_{\text {tol }}$, leading to

$$
\forall k \Delta x \in[0, \pi], \quad \alpha_{\text {loc }}^{d}(\eta, k \Delta x) \leq 1+\alpha_{\text {tol }} .
$$

The previous constraint equation can be reformulated as a single equation:

$$
\left\|\alpha_{\mathrm{loc}}^{d}(\eta, k \Delta x)\right\|_{\infty}=\max _{k \Delta x \in[0, \pi]} \alpha_{\mathrm{loc}}^{d}(\eta, k \Delta x) \leq 1+\alpha_{\mathrm{tol}} .
$$

It has been found that the previous equation is difficult to deal with. However, imposing $\left\|\alpha_{\text {loc }}^{d}(\eta, k \Delta x)\right\|_{\infty}=1+\alpha_{\text {tol }}$ for all $\eta \in[0,1$ [ appears to be more convenient as an equality constraint, even if it is theoretically less flexible than the previous inequality constraint. This formulation strongly modifies the nature of the resulting interpolation method in comparison with noncentered Lagrange or optimized interpolations, for which $\left\|\alpha_{\text {loc }}^{d}(\eta, k \Delta x)\right\|_{\infty}$ is observed to be smaller than $1+\alpha_{\text {tol }}$ at some locations $\eta$. Nevertheless, technical difficulties still remain due to the infinite norm in the resulting equality constraint $\left\|\alpha_{\text {loc }}^{d}(\eta, k \Delta x)\right\|_{\infty}=1+\alpha_{\text {tol }}$, especially if the constraint optimization is expected to be solved with the Lagrange-multiplier technique. In order to make the constraint optimization easier to use, a $L^{2 q}$-norm (where $q \in \mathbb{N}^{*} \backslash\{1\}$ ) can be chosen to approximate the infinite norm, as the $L^{2 q}$-norm converges pointwisely to the infinite norm in $\mathcal{C}^{0}([0, \pi], \mathbb{R})$ for $q \rightarrow+\infty$. The resulting amplification control constraint can be expressed as

$$
\left\|\alpha_{\mathrm{loc}}^{d}(\eta, k \Delta x)\right\|_{2 q}=\left(\int_{0}^{\pi}\left(\alpha_{\mathrm{loc}}^{d}(\eta, k \Delta x)\right)^{2 q} d(k \Delta x)\right)^{1 / 2 q}=1+\alpha_{\mathrm{tol}} .
$$

Copyright $@$ by SIAM. Unauthorized reproduction of this article is prohibited. 
Finally,

$$
\int_{0}^{\pi}\left(\alpha_{\mathrm{loc}}^{d}(\eta, k \Delta x)\right)^{2 q} d(k \Delta x)=\left(1+\alpha_{\mathrm{tol}}\right)^{2 q}
$$

All constraints are now clearly differentiable with respect to the $s_{j}^{d}$ coefficients, which was not evident in the former constraint $\left\|\alpha_{\text {loc }}^{d}(\eta, k \Delta x)\right\|_{\infty}=1+\alpha_{\text {tol }}$. Thus, the resulting constraint optimization can be performed with the Lagrange-multiplier method.

Nevertheless, the choice of $q$ remains a substantial issue. Not only is the integer $q \eta$-dependent, but the nonequivalence between the infinite and $L^{2 q}$ norms makes it impossible to control the approximation error $\varepsilon_{\text {approx }}=\left|\left\|\alpha_{\text {loc }}^{d}\right\|_{2 q}-\left\|\alpha_{\text {loc }}^{d}\right\|_{\infty}\right|$ independently of the function $\alpha_{\text {loc }}^{d}$. In other words, for a given error $\varepsilon_{\text {approx }}, q$ depends on the choice of the interpolation stencil size $n$, the interpolation cell $d$, and the interpolation formal order $p$. It has been numerically observed that $q=30$ ensures a good balance between the approximation accuracy and the convergence of the constraint optimization numerical algorithm which is presented in the next subsection for all tested interpolation methods. The value of $\alpha_{\text {tol }}$ must be taken as small as possible. In most cases, it has been numerically noticed that $\alpha_{\text {tol }}=0.035$ ensures good results. However, some interpolation methods have required a relaxed $\alpha_{\text {tol }}$ of up to 0.3. A relaxed $\alpha_{\text {tol }}$ is chosen essentially for first-cell interpolations, and for stencil sizes greater than 5 , in order to ensure the convergence of the constraint optimization numerical algorithm.

3.1. Constraint optimization algorithm. Let $\mu$ be the extra Lagrange multiplier related to the additional amplification control contraint. The modified Lagrangian function $\mathcal{L}$ is

$$
\begin{aligned}
\mathcal{L}\left(s_{0}^{d}, s_{1}^{d}, \ldots, s_{n-1}^{d}, \lambda_{0}, \ldots, \lambda_{p-1}, \mu\right) & =\int_{\kappa_{l}}^{\kappa_{u}}\left(\varepsilon_{\mathrm{loc}}^{d}(\eta, k \Delta x)\right)^{2} d(k \Delta x) \\
& +\sum_{r=0}^{p-1} \lambda_{r}\left(\sum_{j=0}^{n-1} s_{j}^{d}(j+\chi)^{r}-\delta_{0}^{r}\right) \\
& +\mu\left(\int_{0}^{\pi}\left(\alpha_{\mathrm{loc}}^{d}(\eta, k \Delta x)\right)^{2 q} d(k \Delta x)-\left(1+\alpha_{\mathrm{tol}}\right)^{2 q}\right) .
\end{aligned}
$$

Interpolation coefficients $s_{j}^{d}$ and Lagrange multipliers $\lambda_{r}$ and $\mu$ are found by solving the following nonlinear system: $\forall l \in\{0,1, \ldots, n-1\}$ and $\forall r \in\{0,1, \ldots, p-1\}$,

$$
\frac{\partial \mathcal{L}}{\partial s_{l}^{d}}=0 \quad\left(\mathcal{E}_{l}^{1}\right), \quad \frac{\partial \mathcal{L}}{\partial \lambda_{r}}=0 \quad\left(\mathcal{E}_{r}^{2}\right), \quad \frac{\partial \mathcal{L}}{\partial \mu}=0 \quad\left(\mathcal{E}^{3}\right)
$$

Order constraints are recovered with the $p$ equations $\left(\mathcal{E}^{2}\right)$, and the amplification control constraint is obtained with the equation $\left(\mathcal{E}^{3}\right)$. The $n$ equations $\left(\mathcal{E}^{1}\right)$ are explicitly known: $\forall l \in\{0,1, \ldots, n-1\}$,

$$
\begin{aligned}
& 2 \sum_{j=0}^{n-1} s_{j}^{d}(\eta)\left(\kappa_{u} \operatorname{sinc}(j-l) \kappa_{u}-\kappa_{l} \operatorname{sinc}(j-l) \kappa_{l}\right)-2\left(\kappa_{u} \operatorname{sinc}(l+\chi) \kappa_{u}-\kappa_{l} \operatorname{sinc}(l+\chi) \kappa_{l}\right) \\
+ & \sum_{r=0}^{p-1} \lambda_{r}(\eta)(l+\chi)^{r}+2 q \mu(\eta) \int_{0}^{\pi}\left(\sum_{j=0}^{n-1} s_{j}^{d}(\eta) \cos (j-l) k \Delta x\right)\left(\alpha_{\mathrm{loc}}^{d}(\eta, k \Delta x)\right)^{2 q-2} d(k \Delta x)=0 .
\end{aligned}
$$


A continuation method, as described by Allgower and Georg in [1], could have been used to solve the nonlinear system $\mathcal{E}={ }^{t}\left[\mathcal{E}^{1}, \mathcal{E}^{2}, \mathcal{E}^{3}\right]=0$. For the sake of simplicity, we decided to approximate the solution of the previous nonlinear system with an iterative Newton-Raphson algorithm [24, 26], which is described as follows.

Let $\mathcal{F}$ be the vector of unknowns

$$
\mathcal{F}={ }^{t}\left[s_{0}^{d}(\eta), s_{1}^{d}(\eta), \ldots, s_{n-1}^{d}(\eta), \lambda_{0}(\eta), \lambda_{1}(\eta), \ldots, \lambda_{p-1}(\eta), \mu(\eta)\right] .
$$

Let $\mathcal{F}^{m}$ be the vector of unknowns at the $m$ th iteration. The algorithm is first initialized with an arbitrary starting vector $\mathcal{F}^{0}$. The choice of $\mathcal{F}^{0}$ will be discussed later. The vector of unknowns is then calculated at the $(m+1)$ th iteration from its values at iteration $m$ with the following recurrence relationship:

$$
\mathcal{F}^{m+1}=\mathcal{F}^{m}-\nabla \mathcal{E}^{-1}\left(\mathcal{F}^{m}\right) \mathcal{E}\left(\mathcal{F}^{m}\right) .
$$

The algorithm is stopped if the Euclidean norm of $\mathcal{F}^{m+1}-\mathcal{F}^{m}$ is below a given tolerance error $\varepsilon_{\text {tol }}$. This stop criterion is weak in that it is not a sufficient condition of convergence. In the following, $\varepsilon_{\text {tol }}$ is taken as $10^{-12}$. The quantity $\nabla \mathcal{E}$ is the $(n+p+1) \times(n+p+1)$ nonsingular symmetric Jacobian matrix of vector $\mathcal{E}$.

The key issue of the calculation is finally the choice of $\mathcal{F}^{0}$. It is well known that the main drawback of the Newton-Raphson method resides in the choice of the initial vector [23]. Sebah and Gourdon [27] reported that the Newton-Raphson method always converges if the chosen initialization is "close enough" to a solution, so that the algorithm convergence is local. In the current case, for a given location $\eta$, values of $s_{j}^{d}$ coefficients and Lagrange multipliers are unknown a priori, making the algorithm initialization particularly delicate to guess. The choice was made to initialize the Newton-Raphson algorithm without any dependence with $\eta$. It was numerically observed that the two following initializations ensure algorithm convergence for tested noncentered optimized interpolations with amplification control: $\forall j \in\{0,1, \ldots, n-1\}, \forall r \in\{0,1, \ldots, p-1\}$ :

$$
\mathcal{F}_{\text {init } 0}^{0}:\left\{\begin{array}{rl}
s_{j}^{d}(\eta) & =0, \\
\lambda_{r}(\eta) & =0, \\
\mu(\eta) & =0,
\end{array} \quad \mathcal{F}_{\text {init } 1}^{0}:\left\{\begin{aligned}
s_{j}^{d}(\eta) & =1 / n, \\
\lambda_{r}(\eta) & =0, \\
\mu(\eta) & =0 .
\end{aligned}\right.\right.
$$

A quad-precision computation was required to cope with very large norms of $\mathcal{F}^{m+1}$ $\mathcal{F}^{m}$ due to algorithm initializations, encountered at the very first iterations for largestencil interpolations. The initialization ensuring fastest convergence has been kept. Computations have shown that the initialization $\mathcal{F}_{\text {inito }}^{0}$ is suitable for small-to-mediumstencil size interpolations, whereas $\mathcal{F}_{\text {init } 1}^{0}$ is appropriate for large-stencil size or highorder interpolations. In the following, noncentered optimized interpolations with amplification control are referred to as COI $n \mathrm{p}_{\mathrm{po}}{ }^{d}$, where $n$ is the interpolation stencil size, $p$ the formal order of the interpolation method, and $d$ corresponds to the cell where interpolations are performed.

3.2. Numerical results. Figure 7 shows the 1 st cell interpolation error $\varepsilon^{1}$ for different 6-node noncentered interpolations with amplification control, from 1st to 5 th formal order, and without amplification control. The interpolation error for interpolations with amplification control is close to 2 for high wavenumbers, but decreases quickly for low wavenumbers. It can also be observed that as expected, when the 
formal interpolation order increases, the interpolation error decreases more rapidly. The interpolation error for an optimized interpolation scheme with amplification control is several orders of magnitude larger than the interpolation error of its associated optimized interpolations without amplification control, as depicted in Figure 7 for the 6-point optimized interpolation with amplification control $\left(\mathrm{COI} 6 \mathrm{p} 3 \mathrm{o}^{1}\right)$ and for the 6 -point optimized interpolation without amplification control $\left(\mathrm{OI} 6 \mathrm{p} 3 \mathrm{o}^{1}\right)$.

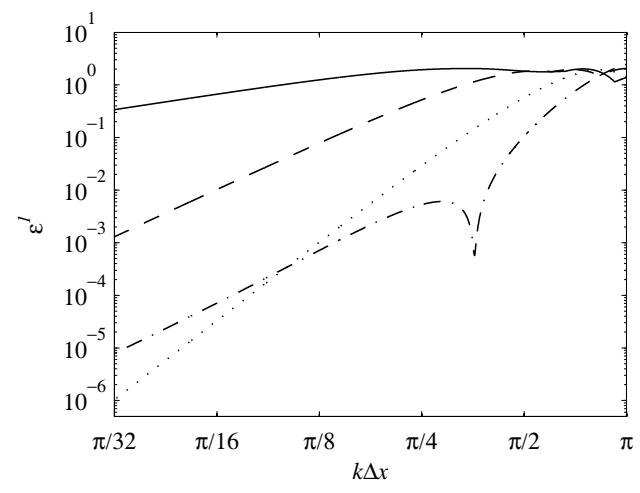

Fig. 7. First-cell interpolation error $\varepsilon^{1}$ as a function of $k \Delta x$ for different orders of 6-point noncentered optimized interpolations with amplitude control, and for the 6-point 3-order noncentered optimized interpolation scheme without amplitude control. $\_$COI6p1o ${ }^{1},---\operatorname{COI} 6 \mathrm{p}^{1} \mathrm{o}^{1}, \cdots \cdots$ COI6p5o ${ }^{1},-\cdots-O I 6 \mathrm{p}^{1}$.

Figure 8(a) exhibits the 1st cell interpolation amplitude amplification $\alpha^{1}$ in the Fourier space for different 6-point noncentered interpolations with amplification control, from 1 st to 5 th formal order. The amplification remains low for all wavenumbers. Amplification amplitude is slightly higher for the 5 th order scheme because a relaxed $\alpha_{\mathrm{tol}}$ of 0.2 was used in the amplification control equation in (11).

Figure 8(b) presents the corresponding 1st cell interpolation phase error $\phi^{1}$. The phase error increases rapidly to $\pi$ for middle-to-high wavenumbers and remains high for high wavenumbers, with several maxima at $\pi$.

(a)

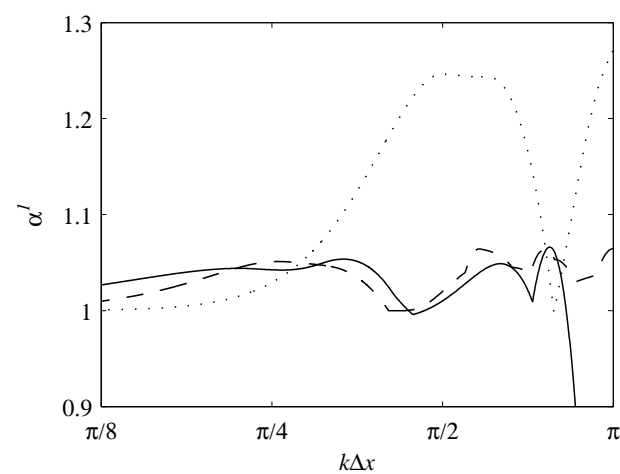

(b)

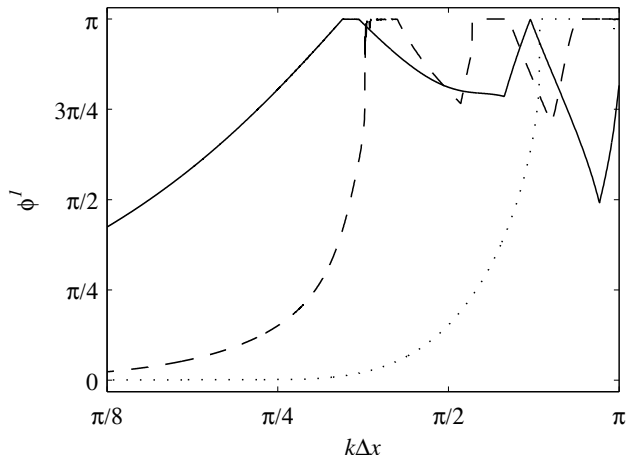

FIG. 8. (a): First cell interpolation amplification $\alpha^{1}$ as a function of $k \Delta x$ for different formal orders of 6-point noncentered interpolations with amplitude amplification control. (b): First-cell interpolation phase error $\phi^{1}$ as a function of $k \Delta x$ for different formal orders of 6-point noncentered interpolations with amplification control. - COI6p1o $1{ }^{1},---$ COI6p3o ${ }^{1}, \cdots \cdot$ COI6p5o ${ }^{1}$. 
(a)

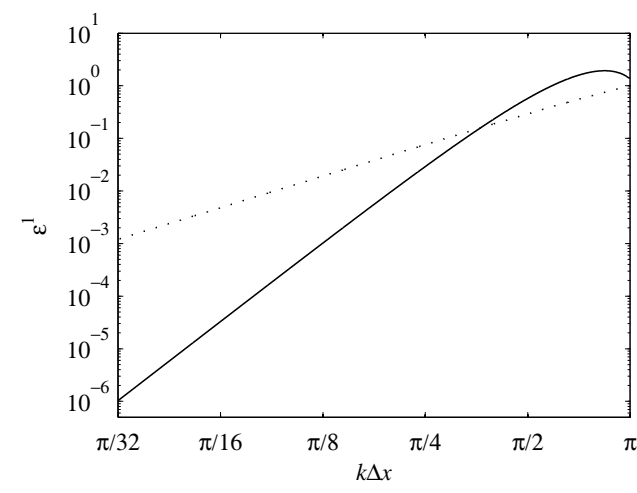

(b)

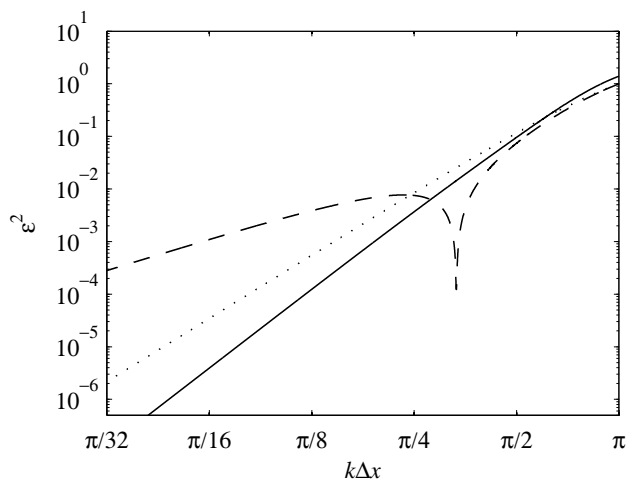

FIG. 9. Comparison with centered interpolations of most suitable noncentered optimized interpolation with amplification control, for first and second cell interpolations. (a): First-cell interpolation error $\varepsilon^{1}$ as a function of $k \Delta x$. COI6p $5 \mathrm{o}^{1}, \ldots \ldots \mathrm{LI} 2 \mathrm{p}^{1}$. (b): Second-cell interpolation error $\varepsilon^{2}$ as a function of $k \Delta x$. COI6p5o $5 \mathrm{o}^{2},---$ OI $4 \mathrm{p}^{2} \mathrm{o}^{2}, \cdots \cdots$ LI4 $\mathrm{p}^{2}$.

A similar study has been performed to focus on the influence of the interpolation stencil size. It has been found that at constant order the interpolation error and amplitude amplification increase with an increase of the stencil size. Thus, in order to choose a suitable interpolation with amplification control, there is a compromise to find between high formal order and low stencil size. An attempt has been performed to choose the most suitable interpolation schemes with amplification control for CAA computations. It suggests the methods ensuring lowest interpolation error in the resolved wavenumber range are the 6-point 5th order noncentered interpolation COI6p5o ${ }^{1}$ for a 1st cell interpolation format, and the 6-point 5 th order noncentered interpolation COI6p5o $\mathrm{o}^{2}$ for a 2nd cell interpolation. Figure 9(a) exhibits the interpolation error for the most suitable first-cell optimized interpolation with amplification control $\left(\mathrm{COI} 6 \mathrm{p} 5 \mathrm{o}^{1}\right)$ and for the nonamplifying centered 2-point Lagrange polynomial interpolation LI $2 \mathrm{p}^{1}$ (linear interpolation). The figure shows that the noncentered interpolation scheme with amplification control ensures a lower interpolation error for resolved wavenumbers than the linear interpolation. The interpolation error for the most suitable second-cell optimized interpolation with amplification control $\left(\mathrm{COI} 6 \mathrm{p} \mathrm{o}^{2}\right)$ and for nonamplifying centered Lagrange and optimized interpolations with a 4-point stencil is depicted in Figure 9(b). Only the 2nd order optimized interpolation is displayed in Figure 9(b), because the 1st and the 3rd order optimized interpolations give almost the same error as the 2nd order optimized interpolation and the 4-point Lagrange interpolation, respectively. For low wavenumbers, the noncentered scheme with amplification control COI6p $5 \mathrm{o}^{2}$ ensures the lowest interpolation error. For wavenumbers higher than $\pi / 4$, the noncentered interpolation with amplification control has an error smaller than that of the centered Lagrange interpolation, but higher than that of the centered optimized interpolation. However, the noncentered method with amplification control COI6p $5 \mathrm{o}^{2}$ is chosen because it ensures the lowest interpolation error in the resolved wavenumbers, except for resolved oscillation wavelengths smaller than 8 mesh nodes where the error remains nevertheless acceptable.

Figure 10 presents the interpolation coefficients of the 1st cell 6-point 5th order interpolation with amplification control COI6p $5 \mathrm{o}^{1}$ as a function of the interpolation location $\eta$. The coefficients are continuous functions of $\eta$, but they exhibit sharp 
variations. High-order piecewise polynomial regressions are performed. The $[0,1[$ interval can be split into $N_{i}$ intervals $\mathcal{I}_{r}\left(r \in\left\{0, \ldots, N_{i}-1\right)\right\}$. Thus, for all intervals $\mathcal{I}_{r}$, the $s_{j}^{d}(\eta)$ interpolation coefficients can be approximated by a polynomial of $\eta$ in $\mathcal{I}_{r}, \operatorname{noted} \widetilde{s}_{j}^{d}(\eta)$, as

$$
\forall j \in\{0,1, \ldots, n-1\}, \forall \eta \in \mathcal{I}_{r}, \widetilde{s}_{j}^{d}(\eta)=\sum_{q=0}^{Q_{r}} a_{q}^{r} \eta^{q} .
$$

For all intervals $\mathcal{I}_{r}$, the $a_{q}^{r}$ coefficients are calculated with the least squares method. Moreover, the polynomial degrees $Q_{r}$ are chosen to ensure a norm of residuals smaller than $5 \times 10^{-3}$ over the whole [0,1[ interval: $\left(\int_{0}^{1}\left(\widetilde{s}_{j}^{d}(\eta)-s_{j}^{d}(\eta)\right)^{2} d \eta\right)^{1 / 2} \leq 5 \times 10^{-3}$. Appendix A provides all the regression coefficients for the two noncentered interpolation methods COI6p5o ${ }^{1}$ (see Table 2) and COI6p5o (see Table 3). Many significant figures were required in the regression coefficients in order to obtain the expected accuracy on the interpolation coefficients $\widetilde{s}_{j}^{d}$.

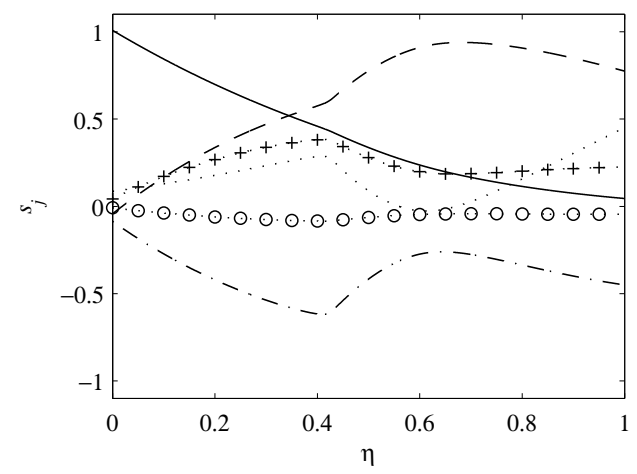

FIG. 10. Interpolation coefficients $s_{j}$ as a function of $\eta$ for the first-cell 6-point 5th order interpolation with amplification control COI6p50 ${ }^{1} \cdot-s_{0},---s_{1}, \cdots s_{2},-\cdots s_{3}, \cdots+\cdot s_{4}$, $\cdots \odot s_{5}$.

The extra constraint added in the method construction has made it possible to control interpolation-related amplification. Nevertheless, even if the amplification is significantly reduced compared with noncentered Lagrange or optimized interpolations, the amplification is not totally removed. It is therefore relevant to look into the time stability of those interpolations in a practical context, i.e., in combination with a low-dispersion, low-dissipation finite-difference scheme, a selective filter, and a Runge-Kutta time integration algorithm.

4. Time stability of optimized interpolations with amplification control. In this section, the 1-D convection equation is considered. The equation to be solved is $\left(\forall x \in \mathcal{I}, \forall t \in \mathbb{R}^{+}\right)$

$$
\left\{\begin{array}{l}
\frac{\partial u}{\partial t}+c_{0} \frac{\partial u}{\partial x}=0 \quad(t>0), \\
u(x, 0)=f_{k}(x)=e^{i k x+i \phi},
\end{array}\right.
$$

where $c_{0}$ is a constant. The initial perturbation simply propagates at the speed $c_{0}$, and the theoretical solution is given by $u_{\mathrm{th}}(x, t)=e^{i k\left(x-c_{0} t\right)+i \phi}$. 
The convection equation is solved on the 1-D multidomain $\mathcal{I}$. The multidomain consists of two uniform meshes $\mathcal{M}$ and $\mathcal{M}^{\prime}$ of same spacing $\Delta x$ with, respectively, $N$ and $N^{\prime}$ nodes. The quantity $x_{0}$ is the location of the first node of the mesh $\mathcal{M}$. The numerical solution is noted $u$ on $\mathcal{M}$ and $u^{\prime}$ on $\mathcal{M}^{\prime}$. The communication from $\mathcal{M}$ to $\mathcal{M}^{\prime}$ is performed with a noncentered $d$-cell $n$-point $p$ th order interpolation with amplification control COI $n \mathrm{p} p \mathrm{o}^{d}$, or without amplification control OInp $p \mathrm{o}^{d}$, whereas the communication from $\mathcal{M}^{\prime}$ to $\mathcal{M}$ is performed with the centered (hence nonamplifying) 8-point optimized interpolation of 2 nd order OI8p $2 \mathrm{o}^{4}$, so that these interpolations generate negligible errors compared to the tested noncentered methods. The mesh $\mathcal{M}^{\prime}$ is translated from $\mathcal{M}$ so that noncentered interpolations are performed at the location $\eta \Delta x$ in the interpolation cell $(\eta \in[0,1[)$. The two meshes are constructed to limit in the superposition zone the number of free nodes $\left(n_{z}\right.$ for the mesh $\mathcal{M}$ and $n_{z}^{\prime}$ for the mesh $\mathcal{M}^{\prime}$ ) that are neither interpolation receiver nodes nor donor nodes. The spatial derivatives are approximated using an 11-point optimized finite-difference scheme (FDo11p) of 4th formal order [5]. The scheme has been optimized in wavenumber space to minimize relative error on the modified wavenumber for wavenumbers up to $k \Delta x=\pi / 2$. Time integration is performed on the interior of each mesh with an explicit 6-stage Runge-Kutta algorithm developed in [5], which is of order 4 for linear problems. The algorithm is optimized in wavenumber space to minimize both dispersion and dissipation for angular frequencies up to $\omega \Delta t=\pi / 2$, and stability is ensured for $\omega \Delta t$ up to around $5 \pi / 4$. The very low dissipation exhibited by these numerical algorithms means that high-wavenumber numerical instabilities of various origins are not significantly damped [22]. In the present computation, these unwanted oscillations are removed with a low-pass filter [5]. To this end, an 11-point 6th order optimized highly selective filtering (SFo11p) is used on the interior of each mesh at every time step $\Delta t$ [6], and applied using the formula $u^{f}\left(x_{i}\right)=u\left(x_{i}\right)-\sigma \sum_{j=-5}^{j=5} d_{j}\left(u\left(x_{i}+j \Delta x\right)-\bar{u}\left(x_{i}+j \Delta x\right)\right)$, where $\sigma$ is called the filtering or dissipation strength. The quantity $u^{f}$ is the function $u$ after filtering, $\bar{u}$ is the time average of $u, d_{j}$ are the symmetric coefficients of the SFo11p scheme, and $x_{i}$ is an interior point. Such a selective filter exhibits a dissipation inferior to $5 \times 10^{-3}$ for wavenumbers up to $k \Delta x=\pi / 2$, which increases rapidly to 1 for grid-to-grid oscillations.

Time update of the receiver nodes is performed by interpolation. When the interpolation process is performed, all receiver points must consequently receive data that has already been integrated in time. Thus, the donor zone and the receiver zone of each mesh must not overlap. Hence, at least one free node is imposed for each mesh of the multidomain. Geometric parameters that guarantee a minimized superposition zone to test the stability of a noncentered interpolation with amplification control are summarized in Table 1. Figure 11 describes the functions of all nodes in a minimal superposition zone. Theoretical values of the solution are imposed for all boundary nodes and for all time steps.

TABLE 1

Multidomain geometric parameters ensuring a minimal superposition zone.

\begin{tabular}{l|cc}
\hline \multicolumn{3}{c}{$\operatorname{Mesh} \mathcal{M}$} \\
\hline$N$ & $n+n_{z}+14$ & \\
$n_{z}$ & 1 & if $n-d \geq 4$ \\
& $4-n+d+n_{z}^{\prime}$ & if $n-d<4$ \\
$x_{0}$ & arbitrary & \\
\hline
\end{tabular}

\begin{tabular}{c|cc}
\hline \multicolumn{3}{c}{ Mesh $\mathcal{M}^{\prime}$} \\
\hline$N^{\prime}$ & $n_{z}^{\prime}+22$ & \\
$n_{z}^{\prime}$ & $n-d-4+n_{z}$ & if $n-d \geq 4$ \\
$x_{0}^{\prime}$ & $x_{0}+(d-1+\eta+5) \Delta x$ & \\
\hline
\end{tabular}




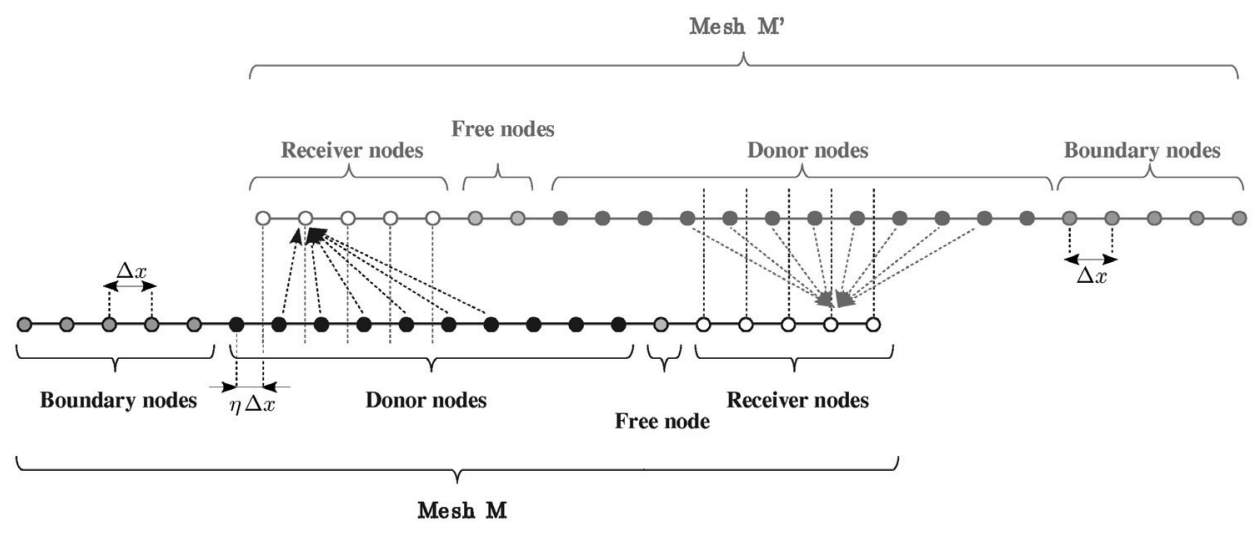

FIG. 11. Detail of all node functions in a minimal superposition zone, with $n=6$ and $d=1$.

To solve the linear problem, four square matrices can be constructed, respectively: $\mathcal{M}_{\text {INT }}$ for interpolations, $\mathcal{M}_{\mathrm{FD}}$ for spatial derivatives, $\mathcal{M}_{\mathrm{TI}}$ for time integration, and $\mathcal{M}_{\mathrm{SF}}$ for filtering. Note that the explicit 6-stage Runge-Kutta time integration is written in the linear case in a matrix form as

$$
\mathcal{M}_{\mathrm{TI}}=\mathcal{I}_{\mathrm{TI}}+\sum_{j=1}^{6} \gamma_{j}(-\mathrm{CFL})^{j}\left(\mathcal{M}_{\mathrm{FD}} \mathcal{M}_{\mathrm{INT}}\right)^{j},
$$

where $\left(\gamma_{j}\right)_{j=1, \ldots, 6}$ are the coefficients of the time integration algorithm [5]. $\mathcal{I}_{\mathrm{TI}}$ is the $\left(N+N^{\prime}\right) \times\left(N+N^{\prime}\right)$ diagonal matrix defined as

$$
\mathcal{I}_{\mathrm{TI}_{j, j}}= \begin{cases}1 & \text { for } 6 \leq j \leq N+N^{\prime}-5 \\ e^{-i \mathrm{CFL} k \Delta x} & \text { otherwise }\end{cases}
$$

where CFL is the Courant-Friedrichs-Lewy constant, defined as CFL $=c_{0} \Delta t / \Delta x$ [11]. It can be noticed in (15) that theoretical values of the solution for all boundary nodes are not assumed for all Runge-Kutta substages, but only for all time steps. Simulations have shown that this simplification tends to generate a very small phase error of the computed solution compared with the analytical solution. However, it showed only a very limited impact on the time stability of the tested noncentered interpolation method, so that it can be ignored in what follows.

An iteration consists of a time integration, followed by an interpolation, and a filtering operation. Hence, if the vector of unknowns ${ }^{t}\left[u_{0}, u_{1}, \ldots, u_{N-1}, u_{0}^{\prime}, u_{1}^{\prime}, \ldots, u_{N^{\prime}-1}^{\prime}\right]$ is noted $\mathcal{U}^{m}$ at time $m \Delta t$, then $\mathcal{U}^{m+1}$ can be explicitly calculated thanks to the following recursive equation:

$$
\mathcal{U}^{m+1}=\mathcal{M}_{\text {iter }} \mathcal{U}^{m}
$$

where $\mathcal{M}_{\text {iter }}=\mathcal{M}_{\mathrm{SF}} \mathcal{M}_{\mathrm{INT}} \mathcal{M}_{\mathrm{TI}}$.

The matrix $\mathcal{M}_{\text {iter }}$ is found to be a diagonalizable matrix, as its associated matrix of eigenvectors has full rank. The stability of the tested noncentered $d$-cell $n$-point $p$ th order interpolations with amplification control COInp $p \mathrm{o}^{d}$ depends consequently on the eigenvalues of the matrix $\mathcal{M}_{\text {iter }}$. The superposition zone remains stable in 
time if each eigenvalue of $\mathcal{M}_{\text {iter }}$ has its modulus smaller than or equal to 1 for all interpolation locations in $[0,1[$ and for all wavenumbers $k \Delta x$ in $[0, \pi]$. A stability $\Lambda$-criterion can be then expressed as

$$
\Lambda(\sigma, \mathrm{CFL})=\max _{\substack{\nu \in \mathrm{Spec}_{\mathcal{M}_{\text {iter }}} \in \in[0,1] \\ k \Delta x \in[0, \pi]}}|\nu(\eta, k \Delta x, \sigma, \mathrm{CFL})| \leq 1 .
$$

Thus, the time stability depends only on the CFL number and the filtering strength $\sigma$. The results of the time stability computations are represented by the map of $\Lambda$ as a function of $(\sigma, \mathrm{CFL})$ for the 1st cell 8-point 4th-order optimized interpolation method $\left(\mathrm{OI} 8 \mathrm{p} 4 \mathrm{o}^{1}\right)$ in Figure 12(a), and its associated method with amplification control $\left(\mathrm{COI} 8 \mathrm{p} 4 \mathrm{o}^{1}\right)$ in Figure 12(b). The stable regions where $\Lambda \leq 1$ are shown in white and the unstable regions where $\Lambda>1$ are in gray. The neutral stability curve separates the stable from the unstable regions. The CFL number is taken in ]0,2]. A critical CFL number $\mathrm{CFL}_{\text {critical }}$ can be defined as the CFL number for which stability is obtained only for $\sigma=1$. For the OI8p $4 \mathrm{o}^{1}$ scheme, $\mathrm{CFL}_{\text {critical }}$ is 1.67. For a given CFL number in $] 0, \mathrm{CFL}_{\text {critical }}$, and for the considered filtering procedure, the dissipation interval of stability $\left[b_{l}, 1\right]$ is smaller when the CFL number increases, as the lower bound $b_{l}$ is increasing to 1 . For CFL numbers higher than $\mathrm{CFL}_{\text {critical }}$, the interpolation scheme is unconditionally unstable, as shown for OI8p4o ${ }^{1}$ in Figure 12(a). Stability is always ensured for the COI8p $4 \mathrm{o}^{1}$ scheme, for moderate to high values of the dissipation $\sigma$ and for the considered CFL range (Figure 12(b)). Thus, the addition of an amplitude amplification constraint has increased the domain of stability of the noncentered interpolation scheme. As a consequence, amplification control gives access to computations with higher time steps or lower dissipation.

(a)

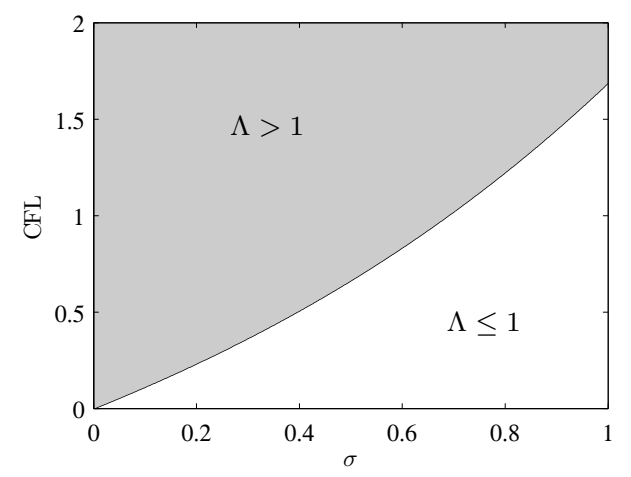

(b)

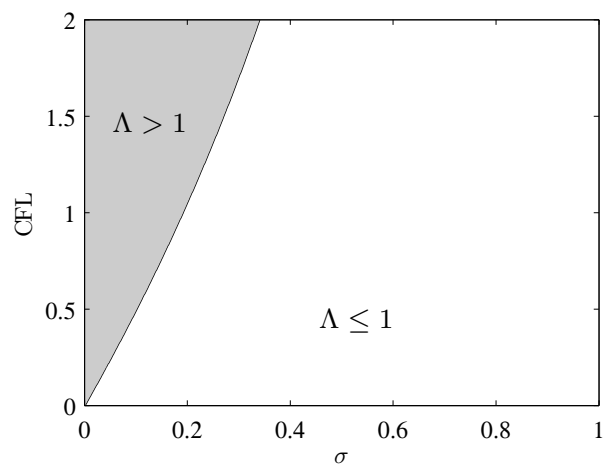

FIG. 12. Stability map of the $\Lambda$-criterion as a function of the filtering strength $\sigma$ and the $C F L$ number. Stable regions where $\Lambda \leq 1$ are in white, unstable regions where $\Lambda>1$ are in gray. (a): OI8p4o $o^{1}$ (b): COI8p4o $o^{1}$ - Neutral stability curve.

5. Validation of noncentered interpolation schemes with amplification control on test cases. In this section, the convection equation is solved numerically on a $1-D$ multidomain $\mathcal{I}$, defined from two uniform meshes $\mathcal{M}$ and $\mathcal{M}^{\prime}$ of same spacing 
$\Delta x: \forall x \in \mathcal{I}, \forall t \in \mathbb{R}^{+}$,

$$
\left\{\begin{array}{l}
\frac{\partial p}{\partial t}+c_{0} \frac{\partial p}{\partial x}=s(x) \quad(t>0), \\
p(x, 0)=g(x)
\end{array}\right.
$$

where $c_{0}$ is the propagation speed taken as $c_{0}=340$, and $g(x)$ is the initial perturbation. The term $s(x)$ is a source term. The analytical solution is

$$
p_{\text {th }}(x, t)=g\left(x-c_{0} t\right)+\frac{1}{c_{0}} \int_{x-c_{0} t}^{x} s(u) d u .
$$

The $\mathcal{M}^{\prime}$ to $\mathcal{M}$ communication is performed with the centered 8-point optimized interpolation of 4 th order OI8p $4 \mathrm{o}^{4}$. The 11-point optimized finite-difference scheme FDo11p is chosen to approximate the spatial derivatives [5], time integration is performed with a 6-step optimized Runge-Kutta algorithm, and an optimized 11-point filter is used. Meshes $\mathcal{M}$ and $\mathcal{M}^{\prime}$ are extended spatially, and a minimal superposition zone is considered (see Figure 11). Only 1st cell 8-point 4th order interpolations without amplification control $\left(\mathrm{OI} 8 \mathrm{p} 4 \mathrm{o}^{1}\right)$ and with amplification control $\left(\mathrm{COI} 8 \mathrm{p} 4 \mathrm{o}^{1}\right)$ will be tested in the following to handle $\mathcal{M}$ to $\mathcal{M}^{\prime}$ noncentered communication. The two meshes $\mathcal{M}$ and $\mathcal{M}^{\prime}$ are translated so that noncentered interpolations are performed at location $\eta_{i} \Delta x$ in the interpolation cell. The parameter $\eta_{i}$ is taken as $\eta_{i}=0.3878$, which ensures the highest interpolation amplification for the noncentered interpolation scheme without amplification control for grid-to-grid oscillations. The mesh $\mathcal{M}$ contains 40101 nodes, and the mesh $\mathcal{M}^{\prime}$ is made of 39917 nodes. The lower bound $x_{0}^{\prime}$ of mesh $\mathcal{M}^{\prime}$ is translated from the mesh $\mathcal{M}$ upper bound $x_{\max }$ by $17-\eta_{i}$ mesh steps, so that $x_{0}^{\prime}=x_{\max }-\left(17-\eta_{i}\right) \Delta x$. The parameters $\Delta x$ and $x_{0}$ are chosen arbitrarily.

In order to convect a low-wavenumber wave packet in the multidomain, the initial perturbation is expressed as

$$
g:\left\{\begin{array}{l}
g(x)=p_{0}+\mathcal{A} \cos \left(\frac{2 \pi\left(x-x_{c}\right)}{a \Delta x}\right) \exp \left(-\ln 2\left(\frac{x-x_{c}}{b \Delta x}\right)^{2}\right) \quad \text { on } \mathcal{M}, \\
g\left(x^{\prime}\right)=p_{0}+\mathcal{A} \cos \left(\frac{2 \pi\left(x^{\prime}-x_{c}\right)}{a \Delta x}\right) \exp \left(-\ln 2\left(\frac{x^{\prime}-x_{c}}{b \Delta x}\right)^{2}\right) \quad \text { on } \mathcal{M}^{\prime}
\end{array}\right.
$$

where $p_{0}$ is the initial mean perturbation, taken as $p_{0}=101325, \mathcal{A}=100$ is the amplitude of the initial perturbation, $x_{c}$ is the initial position of the wave packet, taken as $x_{c}=-600 \Delta x, a=30$ is the number of grid nodes per oscillation wavelength, and $b=20$ is the number of nodes in the half width of the initial wave packet.

The source term is chosen to simulate a numerical noise, which appears in practical computations due to boundary conditions, mesh stretching, or truncation errors in the numerical methods. This numerical noise exhibits typically very small amplitudes compared with the physical signal, and has strong high-wavenumber components. Thus, the source term is taken as a small-amplitude high-wavenumber harmonic noise:

$$
s:\left\{\begin{array}{cc}
s(x)=\frac{2 \pi \mathcal{A} c_{0}}{\lambda_{\mathrm{s}} \Delta x} \mathcal{A}_{\mathrm{s}} \sin \left(2 \pi \frac{x}{\lambda_{\mathrm{s}} \Delta x}\right) & \text { on } \mathcal{M}, \\
s\left(x^{\prime}\right)=\frac{2 \pi \mathcal{A} c_{0}}{\lambda_{\mathrm{s}} \Delta x} \mathcal{A}_{\mathrm{s}} \sin \left(2 \pi \frac{x^{\prime}}{\lambda_{\mathrm{s}} \Delta x}\right) & \text { on } \mathcal{M}^{\prime},
\end{array}\right.
$$

Copyright $@$ by SIAM. Unauthorized reproduction of this article is prohibited. 
where $\lambda_{\mathrm{s}}$ is the number of nodes per oscillation wavelength of the noise source, and $\mathcal{A}_{\mathrm{s}}$ is the ratio of the amplitude of the noise source component in the analytical solution and the wave packet amplitude. Grid-to-grid noise oscillations are considered, so that $\lambda_{\mathrm{s}}=2$. The parameter $\mathcal{A}_{\mathrm{s}}$ is taken as $\mathcal{A}_{\mathrm{s}}=10^{-5}$ so that the noise component amplitude is five orders of magnitude smaller than the convected wave packet amplitude. In what follows, the filtering strength parameter is taken as $\sigma=0.8$.

5.1. Computation at low CFL number: CFL $=\mathbf{0 . 6 7}$. Figure 13 presents the analytical solution of the problem, and its computed solution on the two meshes, at different times and for the two tested noncentered interpolations with and without amplification control and for a CFL $=0.67$. It shows in both cases that the highwavenumber noise source is not amplified in time. The low-wavenumber wave packet is properly convected throughout the superposition zone, and it is correctly recovered on the mesh $\mathcal{M}^{\prime}$ for both methods. Both computations remain stable.
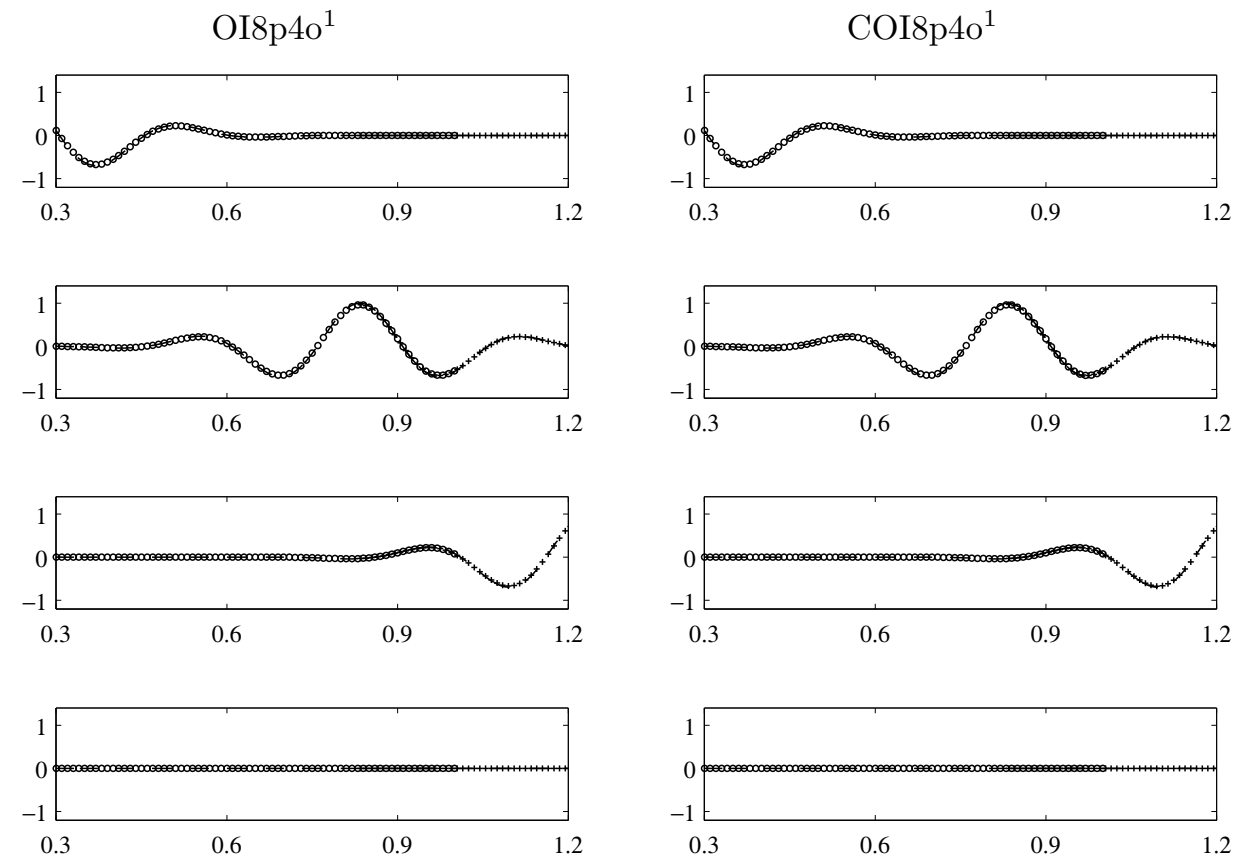

FIG. 13. Test case with $C F L=0.67$. Dimensionless amplitude of the wave packet oscillations $\left(p-p_{0}\right) / \mathcal{A}$ at different times: 960 $\Delta t, 1050 \Delta t, 1110 \Delta t, 3000 \Delta t .---$ Analytical solution, oо о Numerical solution on mesh $\mathcal{M},+++$ Numerical solution on mesh $\mathcal{M}^{\prime}$. Left: First-cell 8-point 4th order optimized interpolation without amplification control. Right: First cell 8-point 4th order optimized interpolation with amplification control.

5.2. Computation at high CFL number: CFL $=1.33$. We now present computations for a high CFL number. Figure 14 displays the analytical solution and the computed solutions on the multidomain, at different times, for a CFL number taken as $\mathrm{CFL}=1.33$. The noncentered interpolation scheme without amplification control OI8p4o ${ }^{1}$ is not stable in time. Indeed, the source oscillations, exhibiting a wavelength of 2 nodes, are amplified at every time step in cells where noncentered interpolations are performed ( $\mathcal{M}$ to $\mathcal{M}^{\prime}$ communication). This amplification is then convected to cells where the $\mathcal{M}^{\prime}$ to $\mathcal{M}$ communication is performed, and spreads 

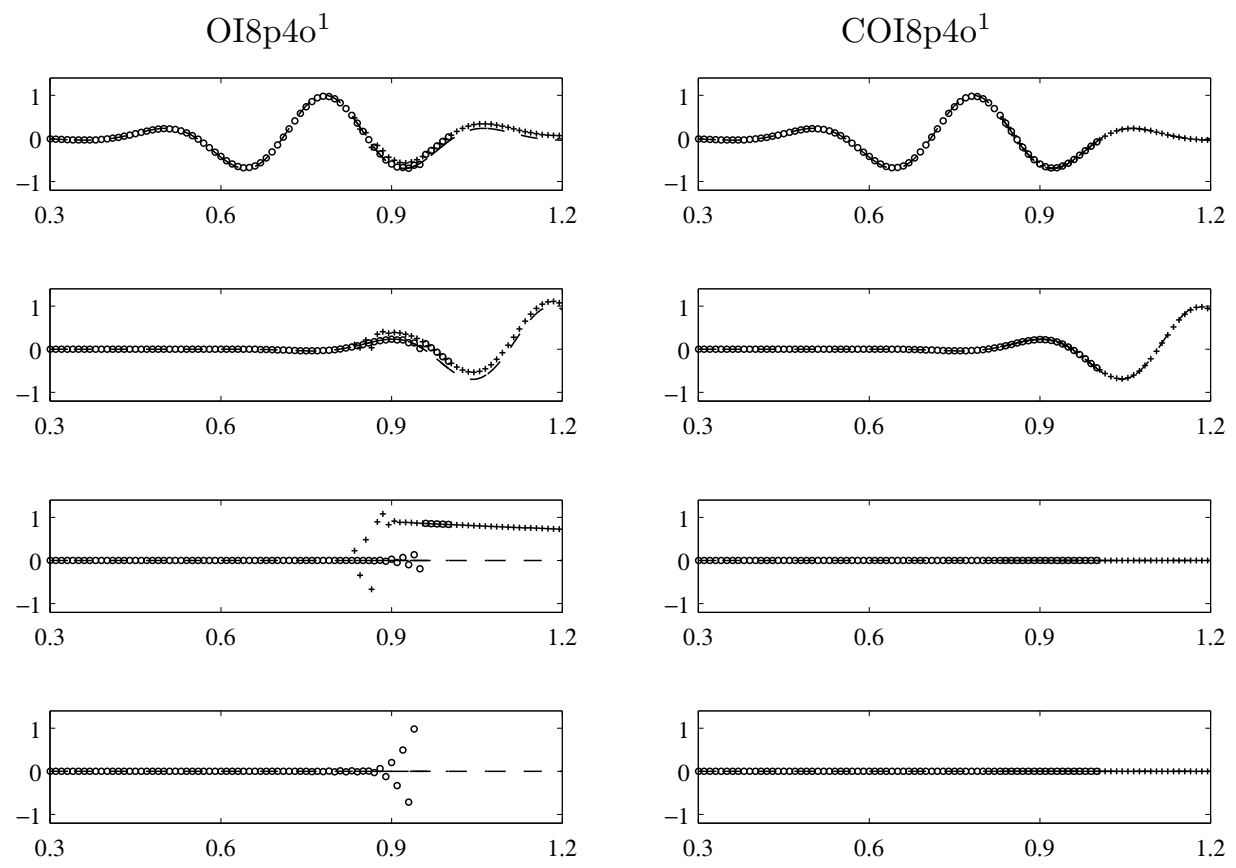

FIG. 14. Test case with $C F L=1.33$. Dimensionless amplitude of the wave packet oscillations $\left(p-p_{0}\right) / \mathcal{A}$ at different times: $540 \Delta t, 570 \Delta t, 750 \Delta t, 960 \Delta t$. --- Analytical solution, o o o Numerical solution on mesh $\mathcal{M},+++$ Numerical solution on mesh $\mathcal{M}^{\prime}$. Left: First-cell 8-point 4th order optimized interpolation without amplification control. Right: First-cell 8-point 4th order optimized interpolation with amplification control.

back to the $\mathcal{M}$ to $\mathcal{M}^{\prime}$ interpolation zone, which self-maintains the amplification phenomenon. In spite of the very small amplitudes of the source term, which are 5 orders of magnitude smaller than the wave packet amplitude, the oscillations generated by noncentered interpolations exhibit a magnitude comparable to the amplitude of the convected perturbation after only 570 iterations. The increasing node-to-node oscillations pollute the convection of the wave packet in the superposition zone, so that the initial perturbation is badly interpolated to the mesh $\mathcal{M}^{\prime}$. Finally, the wave packet is completely flooded by the numerical error coming from the superposition zone after 1500 iterations. On the contrary, the noncentered interpolation scheme with amplification control COI8p $4 \mathrm{o}^{1}$ is stable, and the convected signal remains computed with high fidelity with respect to the analytical solution. A calculation of the $\Lambda$-criterion shows that $\Lambda=1$ for the noncentered interpolation scheme with amplification control COI8p $4 \mathrm{o}^{1}$, and $\Lambda=1.0157>1$ for the noncentered interpolation scheme without amplification control OI8p40 ${ }^{1}$. Hence, the $\Lambda$-criterion correctly predicts the stability of noncentered interpolations with amplification control COI8p $4 \mathrm{o}^{1}$ and indicates as well that the noncentered interpolation method without amplification control OI8p $4 \mathrm{o}^{1}$ is unstable at a CFL number of 1.33 with a filtering strength of 0.8 for some couples $(\eta, k \Delta x)$, which is observed for the current choice of the parameters $\left(\eta=\eta_{i}, k \Delta x=\pi\right)$.

5.3. Progressive decentering. In practical cases, it is advisable to use noncentered optimized interpolations with amplitude control when only one or two nodes are available close to a wall. Indeed, they ensure a lower interpolation error in the resolved wavenumber range than centered optimized or Lagrange interpolations on a 
small interpolation stencil, and they exhibit better time stability than noncentered interpolations without amplification control. Centered optimized interpolation schemes are suitable when the point to be interpolated is located within two points of the wall. The interpolation schemes to be used for a progressive interpolation decentering are summarized in Figure 15.

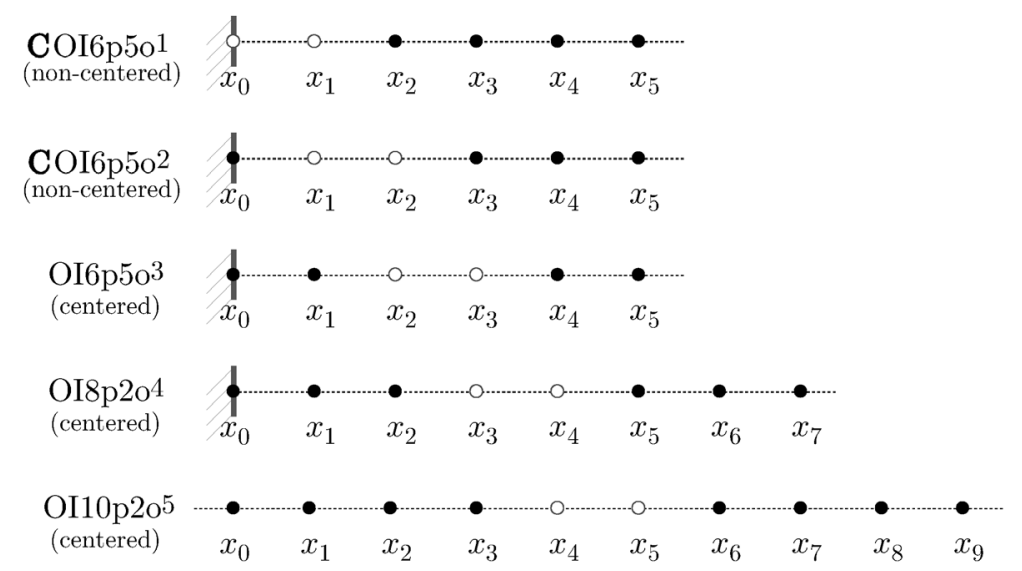

FIG. 15. Interpolation methods to be used for a progressive interpolation decentering. Interpolation stencil is represented in black circles. The interpolation cell is highlighted in white circles. Noncentered interpolations are used when only 1 or 2 nodes are available close to the wall.

6. Concluding remarks. The construction of noncentered high-order optimized interpolation schemes with and without amplification control has been presented in the current work. Noncentered high-order optimized interpolation schemes with amplification control are obtained by solving a minimization problem under constraints related to the interpolation formal order and the amplification control. A survey of the interpolation error for the resulting schemes has been performed to identify the noncentered interpolation schemes ensuring the lowest interpolation error for a given wavenumber range. A criterion based on an eigenvalue study has also been formulated to predict the stability of noncentered interpolation schemes used in combination with a selective filtering removing grid-to-grid oscillations, as a function of the filtering strength and the CFL number. The study reveals that noncentered interpolations without amplification control require smaller CFL numbers and/or higher filtering dissipation to be stable, compared with the noncentered schemes with amplification control. This is a drawback especially in the case of 3-D simulations, where the CFL number should be as large as possible in order to decrease the time needed to complete a simulation. Such a limitation of the CFL number due to stabilization considerations is not required for the presented noncentered interpolation schemes with amplification control, since they can be used in computations with high CFL numbers, on the condition that a selective filtering with a moderate-to-high dissipation be used. These results have been illustrated clearly by test cases.

The work presented here can also be of interest to other fields in which noncentered interpolation techniques are used. It is the case for instance in immersed boundary methods. In addition, the optimization technique with the amplification control that is employed in the design of the high-order noncentered interpolations can be easily adapted for the construction of other stable numerical methods, such as noncentered finite-difference schemes or noncentered filters. 
Appendix A. High-order polynomial regression coefficients.

A.1. COI6p5o ${ }^{1}$. The interval $\left[0,1\left[\right.\right.$ is split into $N_{i}=4$ subintervals $\mathcal{I}_{r}$ defined by $\mathcal{I}_{0}=\left[0, \ell_{1}\left[, \mathcal{I}_{1}=\left[\ell_{1}, \ell_{2}\left[, \mathcal{I}_{2}=\left[\ell_{2}, \ell_{3}\left[\right.\right.\right.\right.\right.\right.$, and $\mathcal{I}_{3}=\left[\ell_{3}, 1\left[\right.\right.$, where $\ell_{1}=0.4113$, $\ell_{2}=0.4279$, and $\ell_{3}=0.6439$.

TABLE 2

$a_{q}^{r}$ coefficients for polynomial regressions of interpolation coefficients $\widetilde{s}_{j}^{1}(\eta)$ for COI6 $55 \mathrm{o}^{1}$.

\begin{tabular}{|c|c|c|c|c|c|}
\hline & & $r=0$ & $r=1$ & $r=2$ & $r=3$ \\
\hline \multirow{6}{*}{$\widetilde{s}_{0}^{1}$} & $a_{0}^{r}$ & 1.00862697955555 & -0.27020537609112 & 1.24821066747851 & 3.11048900577393 \\
\hline & $a_{1}^{r}$ & -1.74605782314864 & 4.53663828255703 & -2.42460153299612 & -13.85570842916752 \\
\hline & $a_{2}^{\frac{1}{r}}$ & 1.07244372290761 & -6.79127228116428 & 1.06786026359128 & 28.10309862851732 \\
\hline & $a_{3}^{r}$ & -0.73323175280584 & - & 0.27291758666731 & -30.45862899241656 \\
\hline & $a_{4}^{r}$ & 1.89635085848184 & - & - & 16.96258279853443 \\
\hline & $a_{5}^{r}$ & -2.38058309311138 & - & - & -3.81683557094936 \\
\hline \multirow{9}{*}{$\widetilde{s}_{1}^{1}$} & $a_{0}^{r}$ & -0.04274527229 & -84.12842233689 & 26.52893763861 & -24.339688469955 \\
\hline & $a_{1}^{r}$ & 2.26907724837 & 617.83887992015 & -315.39442482956 & 165.720547552538 \\
\hline & $a_{2}^{r}$ & -1.03968958084 & -1504.86660841143 & 1539.57068080152 & -455.881862122109 \\
\hline & $a_{3}^{r}$ & -16.64615276921 & 1224.13159195469 & -3937.95673126612 & 674.809326329952 \\
\hline & $a_{4}^{r}$ & 154.75635883076 & - & 5632.58544732633 & -566.187510201081 \\
\hline & $a_{5}^{r}$ & -818.71560092582 & - & -4284.28183106005 & 254.358828590791 \\
\hline & $a_{6}^{r}$ & 2493.15788764139 & - & 1353.17697728287 & -47.704733599092 \\
\hline & $a_{7}^{r}$ & -4036.94621090143 & - & - & - \\
\hline & $a_{8}^{r}$ & 2694.82917904375 & - & - & - \\
\hline \multirow{10}{*}{$\widetilde{s}_{2}^{1}$} & $a_{0}^{r}$ & 0.0856788879 & 168.25942804625 & -25315.9047514 & 48.67937693980 \\
\hline & $a_{1}^{r}$ & 0.4204151743 & -1230.70238855824 & 427590.2323062 & -326.44109510429 \\
\hline & $a_{2}^{\frac{1}{r}}$ & 0.3823261806 & 3005.90459486869 & -3202629.5563575 & 907.84705757517 \\
\hline & $a_{3}^{r}$ & -16.1104826798 & -2447.40306456714 & 13962359.5352392 & -1348.61865265606 \\
\hline & $a_{4}^{r}$ & 288.0050638403 & - & -39047451.2940583 & 1132.29168706536 \\
\hline & $a_{5}^{\frac{4}{r}}$ & -2431.4275273476 & - & 72645330.6600455 & -508.71765717992 \\
\hline & $a_{6}^{r}$ & 11502.1176663545 & - & -89909897.1414153 & 95.40946719785 \\
\hline & $a_{7}^{r}$ & -31195.5176636935 & - & 71383985.3009916 & - \\
\hline & $a_{8}^{r}$ & 45328.6010643753 & - & -32990738.3325973 & - \\
\hline & $a_{9}^{r}$ & -27399.9446721963 & - & 6762125.5959478 & - \\
\hline \multirow{10}{*}{$\widetilde{s}_{3}^{1}$} & $\overline{a_{0}^{r}}$ & -0.0856788879 & -168.26201141860 & 25315.9047514 & -48.67937693977 \\
\hline & $a_{1}^{r}$ & -2.0870818409 & 1229.06035060871 & -427591.8989727 & 324.77442843735 \\
\hline & $a_{2}^{r}$ & 2.0343404861 & -3003.57597291257 & 3202631.9730230 & -905.43039090766 \\
\hline & $a_{3}^{r}$ & 15.2771493466 & 2446.70961189004 & -13962360.3685677 & 1347.78531932136 \\
\hline & $a_{4}^{r}$ & -287.9217305079 & - & 39047451.3773780 & -1132.20835373078 \\
\hline & $a_{5}^{r}$ & 2431.4275273544 & - & -72645330.6600198 & 508.71765717931 \\
\hline & $a_{6}^{r}$ & -11502.1176663828 & - & 89909897.1413830 & -95.40946719773 \\
\hline & $a_{7}^{r}$ & 31195.5176637629 & - & -71383985.3009652 & - \\
\hline & $a_{8}^{r}$ & -45328.6010644672 & - & 32990738.3325846 & - \\
\hline & $a_{9}^{\stackrel{o}{r}}$ & 27399.9446722471 & - & -6762125.5959450 & - \\
\hline \multirow{9}{*}{$\widetilde{s}_{4}^{1}$} & $a_{0}$ & 0.04274527229 & 84.13229739544 & -26.52893763853 & 24.339688469874 \\
\hline & $a_{1}^{r}$ & 1.48092275163 & -614.12582299604 & 319.14442482861 & -161.970547551945 \\
\hline & $a_{2}^{r}$ & -2.83531041916 & 1501.12367547773 & -1543.44568079712 & 452.006862120304 \\
\hline & $a_{3}^{r}$ & 17.89615276918 & -1223.09141293941 & 3939.20673125528 & -673.559326327034 \\
\hline & $a_{4}^{r}$ & -154.88135883049 & - & -5632.71044731136 & 566.062510198441 \\
\hline & $a_{5}^{\frac{4}{r}}$ & 818.71560092445 & - & 4284.28183104907 & -254.358828589521 \\
\hline & $a_{6}^{r}$ & -2493.15788763731 & - & -1353.17697727953 & 47.704733598838 \\
\hline & $a_{7}^{r}$ & 4036.94621089501 & - & - & - \\
\hline & $a_{8}^{r}$ & -2694.82917903962 & - & - & - \\
\hline \multirow{6}{*}{$\widetilde{s}_{5}^{1}$} & $\overline{a_{0}^{r}}$ & -0.00862697955555 & 1.24329715560303 & -0.25152951487045 & -2.11048900577165 \\
\hline & $a_{1}^{r}$ & -0.33727551018475 & -6.42449688600345 & 0.36648044226314 & 11.77237509582031 \\
\hline & $a_{2}^{r}$ & 0.38588961042651 & 7.76908049397958 & 0.31907777431298 & -26.64476529515033 \\
\hline & $a_{3}^{r}$ & 0.31656508613477 & - & -0.60025865410704 & 30.04196232570929 \\
\hline & $a_{4}^{r}$ & -1.85468419180568 & - & - & -16.92091613184341 \\
\hline & $a_{5}^{r}$ & 2.38058309310489 & - & - & 3.81683557094355 \\
\hline
\end{tabular}

Copyright $@$ by SIAM. Unauthorized reproduction of this article is prohibited. 
A.2. COI6p5o ${ }^{2}$. The whole interval $\mathcal{I}_{1}=[0,1[$ is considered.

TABLE 3

$a_{q}^{r}$ coefficients for polynomial regressions of interpolation coefficients $\widetilde{s}_{j}^{2}(\eta)$ for COI6p50 ${ }^{2}$.

\begin{tabular}{|c|c|c|c|c|c|}
\hline \multirow{10}{*}{$\widetilde{s}_{0}^{2}$} & $a_{0}^{1}$ & 0.01267209009992 & \multirow{10}{*}{$\widetilde{s}_{1}^{2}$} & $a_{0}^{1}$ & 0.936964693062 \\
\hline & $a_{1}^{1}$ & -0.17068457340145 & & $a_{1}^{1}$ & -1.259307866391 \\
\hline & $a_{2}^{1}$ & 0.15104114173173 & & $a_{2}^{1}$ & 1.346170123030 \\
\hline & $a_{3}^{1}$ & 0.38008352519153 & & $a_{3}^{1}$ & -8.254813620145 \\
\hline & $a_{4}^{1}$ & -0.91108893260404 & & $a_{4}^{1}$ & 33.163008592779 \\
\hline & $a_{5}^{1}$ & 0.78178521680509 & & $a_{5}^{1}$ & -82.748918037865 \\
\hline & $a_{6}^{1}$ & -0.24238143637078 & & $a_{6}^{1}$ & 130.677134106488 \\
\hline & $a_{7}^{1}$ & - & & $a_{7}^{1}$ & -124.958259741914 \\
\hline & $a_{8}^{1}$ & - & & $a_{8}^{1}$ & 65.426155975770 \\
\hline & $a_{9}^{1}$ & - & & $a_{9}^{1}$ & -14.335452481196 \\
\hline \multirow{10}{*}{$\widetilde{s}_{2}^{2}$} & $a_{0}^{1}$ & 0.126070613876 & \multirow{10}{*}{$\widetilde{s}_{3}^{2}$} & $a_{0}^{1}$ & -0.126070613876 \\
\hline & $a_{1}^{1}$ & 2.351949066116 & & $a_{1}^{1}$ & -1.351949066116 \\
\hline & $a_{2}^{1}$ & -4.109006912726 & & $a_{2}^{1}$ & 4.525673579392 \\
\hline & $a_{3}^{1}$ & 17.176293906942 & & $a_{3}^{1}$ & -17.676293906941 \\
\hline & $a_{4}^{1}$ & -66.409350518852 & & $a_{4}^{1}$ & 66.492683852171 \\
\hline & $a_{5}^{1}$ & 165.497836075735 & & $a_{5}^{1}$ & -165.497836075671 \\
\hline & $a_{6}^{1}$ & -261.354268213205 & & $a_{6}^{1}$ & 261.354268213065 \\
\hline & $a_{7}^{1}$ & 249.916519484272 & & $a_{7}^{1}$ & -249.916519484106 \\
\hline & $a_{8}^{1}$ & -130.852311951889 & & $a_{8}^{1}$ & 130.852311951788 \\
\hline & $a_{9}^{1}$ & 28.670904962494 & & $a_{9}^{1}$ & -28.670904962469 \\
\hline \multirow{10}{*}{$\widetilde{s}_{4}^{2}$} & $a_{0}^{1}$ & 0.063035306938 & \multirow{10}{*}{$\widetilde{s}_{5}^{2}$} & $a_{0}^{1}$ & -0.01267209009992 \\
\hline & $a_{1}^{1}$ & 0.509307866391 & & $a_{1}^{1}$ & -0.07931542659855 \\
\hline & $a_{2}^{1}$ & -2.221170123029 & & $a_{2}^{1}$ & 0.30729219160160 \\
\hline & $a_{3}^{1}$ & 9.004813620136 & & $a_{3}^{1}$ & -0.63008352519156 \\
\hline & $a_{4}^{1}$ & -33.288008592746 & & $a_{4}^{1}$ & 0.95275559927079 \\
\hline & $a_{5}^{1}$ & 82.748918037817 & & $a_{5}^{1}$ & -0.78178521680517 \\
\hline & $a_{6}^{1}$ & -130.677134106500 & & $a_{6}^{1}$ & 0.24238143637081 \\
\hline & $a_{7}^{1}$ & 124.958259742020 & & $a_{7}^{1}$ & - \\
\hline & $a_{8}^{1}$ & -65.426155975875 & & $a_{8}^{1}$ & - \\
\hline & $a_{9}^{1}$ & 14.335452481230 & & $a_{9}^{1}$ & - \\
\hline
\end{tabular}

\section{REFERENCES}

[1] E. Allgower and K. Georg, Simplicial and continuation methods for approximating, fixed points and solutions to systems of equations, SIAM Rev., 22 (1980), pp. 28-85.

[2] J. A. Benek, J. L. Steger, F. C. Dougherty, and P. G. Buning, Chimera: A GridEmbedding Technique, Tech. report AEDC-TR-85-64, Arnold Engineering Development Center, Arnold AFB, TN, 1986.

[3] J. Berland, C. Bogey, O. Marsden, and C. Bailly, High-order, low dispersive and low dissipative explicit schemes for multiple-scale and boundary problems, J. Comput. Phys., 224 (2007), pp. 637-662.

[4] D. P. Bertsekas, Constrained Optimization and Lagrange Multipliers Methods, Athena Scientific, Nashua, NH, 1982.

[5] C. Bogey and C. Bailly, A family of low dispersive and low dissipative explicit schemes for flow and noise computation, J. Comput. Phys., 194 (2004), pp. 194-214.

Copyright (c) by SIAM. Unauthorized reproduction of this article is prohibited. 
[6] C. Bogey, N. De Cacqueray, And C. Bailly, A shock-capturing methodology based on adaptative spatial filtering for high-order non-linear computations, J. Comput. Phys., 228 (2009), pp. 1447-1465.

[7] J. P. Boyd, Chebyshev and Fourier Spectral Methods, Springer-Verlag, New York, 1989.

[8] J. P. Boyd, A fast algorithm for Chebyshev, Fourier, and sinc interpolation onto an irregular grid, J. Comput. Phys., 103 (1992), pp. 243-257.

[9] C. Canuto, M. Y. Hussaini, A. Quarteroni, and T. A. Zang, Spectral Methods in Fluid Dynamics, Springer-Verlag, New York, 1988.

[10] T. Colonius, and S. K. Lele, Computational aeroacoustics: Progress on nonlinear problems of sound generation, Prog. Aerospace Sci., 40 (2004), pp. 345-416.

[11] R. Courant, K. Friedrichs, And H. Lewy, Über die partiellen Differenzengleichungen der mathematischen Physik, Math. Ann., 100 (1928), pp. 32-74.

[12] J. W. Delfs, An Overlapped Grid Technique for High Resolution CAA Schemes for Complex Geometries, in the 39th Aerospace Sciences Meeting and Exhibit, Reno, NV, AIAA paper 2001-2199, 2001.

[13] G. Desquesnes, M. Terracol, E. Manoha, and P. Sagaut, On the use of high order overlapping grid method for coupling in CFD/CAA, J. Comput. Phys., 220 (2006), pp. 355-382.

[14] J. B. Freund, S. K. Lele, And P. Moin, Direct Simulation of a Mach 1.92 Jet and Its Sound Field, in the Fourth AIAA/CEAS Aeroacoustics Conference, Toulouse, France, AIAA paper 1998-2291, 1998.

[15] X. Gloerfelt and P. Lafon, Direct computation of the noise induced by a turbulent flow through a diaphragm in a duct at low Mach number, Comp. Fluids, 37 (2008), pp. 388-401.

[16] K. A. Kurbatskin And C. K. W. TAM, Cartesian Boundary Treatment of Curved Walls for High-Order, Computational Aeroacoustics Finite Difference Schemes, in the 34th Aerospace Sciences Meeting and Exhibit, Reno, NV, AIAA paper 1996-275, 1996.

[17] J. L. Lagrange, Leçons élémentaires sur les mathématiques données à l'École Normale en 1795, Euvres VII, ed. Gauthier-Villars, Paris, France, 1877, pp. 183-287.

[18] T. Le Garrec, X. Gloerfelt, and C. Corre, Multi-size-mesh, multi-time-step algorithm for noise computation around an airfoil in curvilinear meshes, in the 13th AIAA/CEAS Aeroacoustics Conference, Rome, Italy, AIAA paper 2007-3504, 2007.

[19] S. K. LELE, Compact finite difference schemes with spectral-like resolution, J. Comput. Phys., 103 (1992), pp. 16-42.

[20] M. Lummer, J. W. Delfs, And T. Lauke, Simulation of sound generation by vorticies passing the trailing edge of airfoils, in the 8th AIAA/CEAS Aeroacoustics Conference, Breckenridge, CO, AIAA paper 2002-2578, 2002.

[21] O. Marsden, Calcul direct du rayonnement acoustique de profils par une approche curviligne d'ordre élevé, Ph.D. thesis, École Centrale de Lyon, Lyon, France, 2005.

[22] O. Mardden, C. Bogey, And C. Bailly, High-order curvilinear simulations of flows around non-Cartesian bodies, J. Comput. Acoust., 13 (2005), pp. 731-748.

[23] J. R. Mourraille, Traité de la résolution des équations en général, Jean Mossy éd., Marseille, France, 1768.

[24] I. Newton, Methodus fluxionum et serierum infinitarum, (1664-1671).

[25] N. Peller, A. Le Duc, F. Tremblay, and M. Manhart, High-order stable interpolations for immersed boundary methods, Int. J. Numer. Meth. Fluids, 52 (2006), pp. 1175-1193.

[26] J. RAPHSOn, Analysis aequationum universalis, London (1690).

[27] P. Sebah and X. Gourdon, Newton's Method and High Order Iterations, Tech. report, 2001. Available online at http://numbers.computation.free.fr/constants/algorithms/newton. html.

[28] S. E. Sherer and J. N. Scott, Comparison of Highly Accurate Interpolation Methods, in the 39th Aerospace Sciences Meeting and Exhibit, Reno, NV, AIAA paper 2001-0282, 2001.

[29] S. E. Sherer AND J. N. SCOTT, High-order compact finite-difference methods on general overset grids, J. Comput. Phys., 210 (2005), pp. 459-496.

[30] V. Strassen, Gaussian elimination is not optimal, Numer. Math., 13 (1969), pp. 354-356.

[31] C. K. W. TAM AND Z. Dong, Wall boundary conditions for high-order finite-difference schemes in computational aeroacoustics, Theoret. Comput. Fluid Dyn., 6 (1994), pp. 303-322.

[32] C. K. W. TAm And K. A. Kurbatskil, A wavenumber based extrapolation and interpolation method for use in conjuction with high-order finite difference schemes, J. Comput. Phys., 157 (2000), pp. 588-617.

[33] C. K. W. TAm And J. C. WeBB, Dispersion-relation-preserving finite difference schemes for computational acoustics, J. Comput. Phys., 107 (1993), pp. 262-281.

[34] E. Waring, Problems concerning interpolations, Philos. Trans. R. Soc. London, 69 (1779), pp. $59-67$. 